\title{
Stimulus dependence of theta rhythmic activity in primate V1 and its potential relevance for visual perception
}

\author{
Prasakti Tenri Fanyiwi ${ }^{1}$, Beshoy Agayby ${ }^{1}$, Ricardo Kienitz ${ }^{1,2,3}$, Marcus Haag ${ }^{4}$, Michael C. \\ Schmid ${ }^{1,4}$ \\ 1 Biosciences Institute, Newcastle University, Newcastle upon Tyne, United Kingdom; 2 Ernst \\ Strüngmann Institute (ESI) for Neuroscience in Cooperation with Max Planck Society, \\ Frankfurt, Germany; 3 Epilepsy Center, Goethe University, Frankfurt, Germany; 4 Faculty of \\ Science and Medicine, University of Fribourg, Fribourg, Switzerland \\ Correspondence: prasakti.tenri@gmail.com or michael.schmid@unifr.ch
}

\begin{abstract}
A growing body of psychophysical research reports theta $(3-8 \mathrm{~Hz})$ rhythmic fluctuations in visual perception that are often attributed to an attentional sampling mechanism arising from theta rhythmic neural activity in mid- to high-level cortical association areas. However, it remains unclear to what extent such neuronal theta oscillations might already emerge at early sensory cortex like the primary visual cortex (V1), e.g. from the stimulus filter properties of neurons. To address this question, we recorded multi-unit neural activity from V1 of two macaque monkeys viewing a static visual stimulus with variable sizes, orientations and contrasts. We found that among the visually responsive electrode sites, more than $50 \%$ showed a spectral peak at theta frequencies. Theta power varied with varying basic stimulus properties. Within each of these stimulus property domains (e.g. size), there was usually a single stimulus value that induced the strongest theta activity. In addition to these variations in theta power, the peak frequency of theta oscillations increased with increasing stimulus size and also changed depending on the stimulus position in the visual field. Further analysis confirmed that this neural theta rhythm was indeed stimulus-induced and did not arise from small fixational eye movements (microsaccades). When the monkeys performed a detection task of a target embedded in a theta-generating visual stimulus, reaction times also tended to fluctuate at the same theta frequency as the one observed in the neural activity. The present study shows that a highly stimulus-dependent neuronal theta oscillation can be elicited in V1 that appears to influence the temporal dynamics of visual perception.
\end{abstract}

\section{Introduction}

Although our experience of the world seems continuous, the underlying perceptual processes may be discrete or rhythmic ${ }^{1,2}$. Accordingly, our brain might periodically sample and process information from the outside world. In the domain of vision, a number of studies report supporting evidence for this view of rhythmic perceptual sampling. When subjects view images of natural scenes or perform search or reading tasks, saccadic eye movements are performed roughly every $200-300 \mathrm{~ms}$, i.e. in the theta $(3-8 \mathrm{~Hz})$ frequency range ${ }^{3,4}$. More recently, researchers have started to investigate whether similar rhythmicity can also be 
observed during visual detection tasks that require subjects to hold their gaze stable, while covertly orienting their attention to visual objects placed across different positions of the visual scene. During such attention-demanding tasks, subjects regularly display small fixational eye movements, termed microsaccades, which tend to occur also in the theta frequency range ${ }^{5-7}$. In addition to microsaccadic assessment, several research groups have developed paradigms to assess rhythmicity during cognitive task performance ${ }^{8-13}$ : By systematically assessing the time course of target detection performance and reaction times, these studies collectively reported a waxing and waning of performance across times in the theta frequency range. Taken together, these findings have been interpreted as supporting evidence for the hypothesis that the brain may carry out rhythmic sampling of visual stimuli $14-16$.

While new behavioural investigations continue delineating the larger context of such sampling under different task conditions, electrophysiological studies have started to identify the neural correlates of rhythmic task performance and to characterise the conditions that lead to theta rhythmic neuronal activity. A prevailing view is that rhythmic sampling arises from high-level brain areas engaged in attentional control. Evidence from human EEG/MEG recordings consistently shows the dependence of performance on the phase of theta oscillations in the frontoparietal areas ${ }^{17-21}$. Recent intracortical studies in monkeys ${ }^{22-25}$ and humans ${ }^{26}$ confirmed this correlation between theta rhythmic neural activity in frontoparietal areas and pulvinar with behavioural performance. Taken together, the results from these studies appear to point to a source of theta rhythmic neural activity in areas whose function has been linked with attentional control ${ }^{27}$.

However, theta oscillations are not limited to higher brain areas ${ }^{28}$. There are reports of theta oscillations in the visual association cortex such as temporal lobe regions ${ }^{29-32}$ and area $\mathrm{V} 4{ }^{33-35}$, in particular when multiple visual stimuli are presented and subjects need to distribute their attention accordingly resulting in rhythmic sampling. In addition to these findings in higher-order areas, very recent findings point to the existence of theta oscillations at an even earlier level, the primary visual cortex (V1); theta-rhythmic microsaccades might reflect or induce rhythmic brain activity in V1 and influence inter-cortical processing ${ }^{5,6}$. Using Granger causality, a statistical method for determining temporal precedence, it has been demonstrated that theta measured in the context of a visual attention task mainly flows in feedforward direction from V1 to higher-level cortical areas, such as V4 ${ }^{36,37}$. Consistent with this finding is the observation that removal of $\mathrm{V} 1$ eliminates theta, but not gamma rhythmic neural responses in $\mathrm{V}^{3}{ }^{38}$. However, it remains unknown how theta rhythmic activity might emerge from the processing of V1 neurons.

The responses of $\mathrm{V} 1$ neurons to visual stimulation are well known to reflect basic stimulus properties such as orientation, spatial frequency, contrast, and size ${ }^{39}$. When tested with a drifting grating stimulus, many $\mathrm{V} 1$ neurons exhibit responses that are preferentially tuned to temporal frequencies in the theta range ${ }^{40,41}$. An interesting hypothesis is therefore that V1 neurons might act as a theta-tuned temporal filter to incoming sensory information thereby possibly providing a perceptual sampling mechanism. Accordingly, presenting a static visual stimulus could induce theta oscillations, consistent with the preferred frequency of many V1 neurons.

To test this hypothesis, we presented static stimuli varying in three properties known to modulate $\mathrm{V} 1$ responses, namely size, contrast, and orientation. Following characterisation of how visual stimulus parameters are linked to the emergence of theta oscillations in V1, we 
bioRxiv preprint doi: https://doi.org/10.1101/2021.11.30.470367; this version posted December $1,2021$. The copyright holder for this preprint (which was not certified by peer review) is the author/funder, who has granted bioRxiv a license to display the preprint in perpetuity. It is made available under aCC-BY-NC-ND 4.0 International license.

also examined to what extent these V1 oscillations might be correlated with the occurrence of microsaccades and theta-rhythmic reaction time (RT) fluctuations during visual detection.

\section{Results}

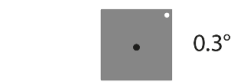

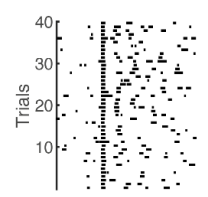<smiles>CC(C)CC(C)C</smiles>

B
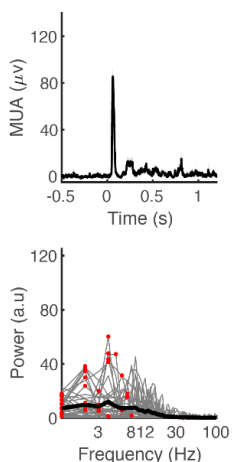
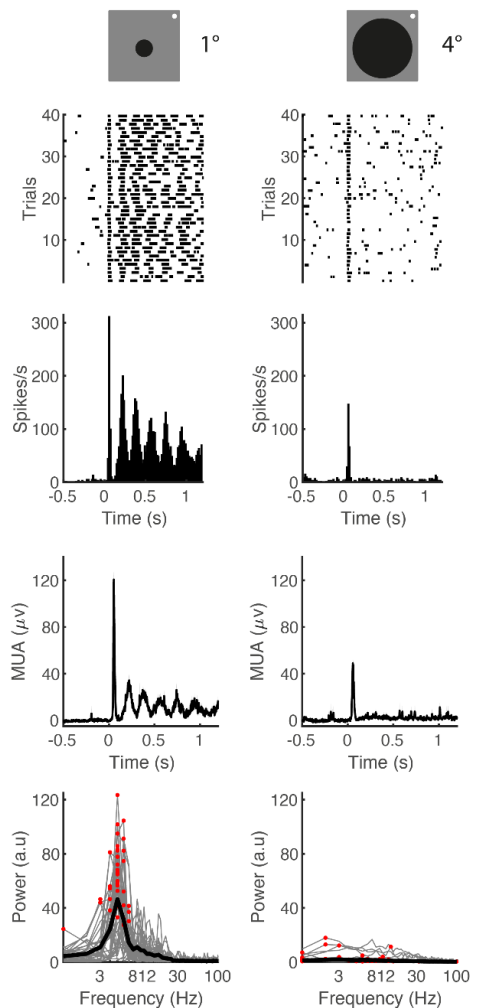

Monkey AL

D

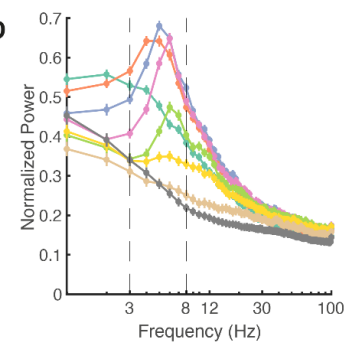

E

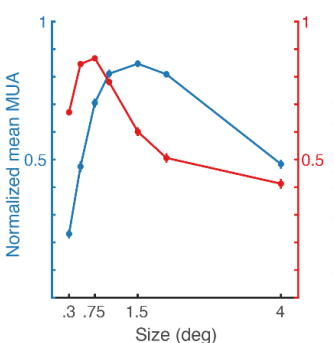

$\mathbf{F}$

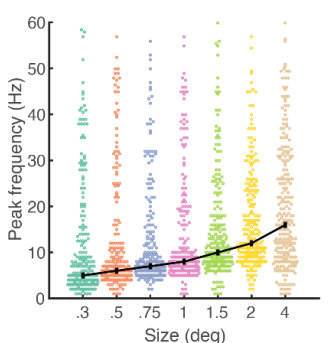

Monkey DP
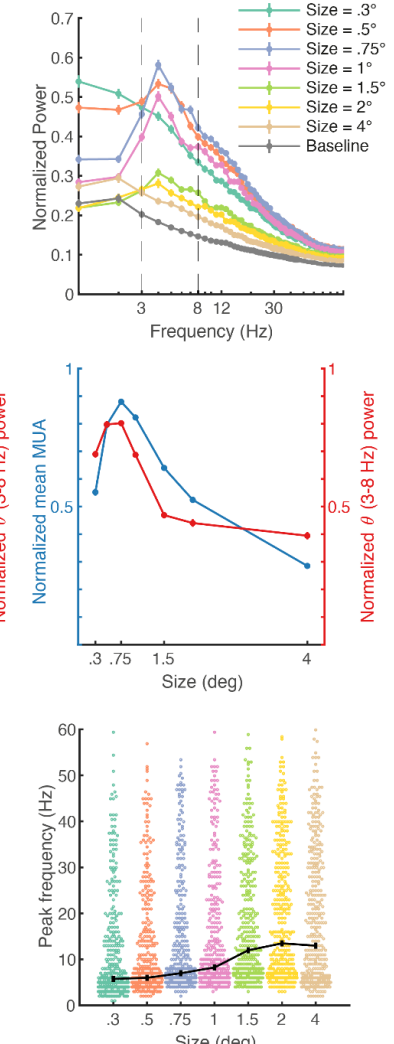

Figure 1. Theta oscillations at different stimulus sizes

(A) Top: Schematic of the stimulus, a black disk, presented at three different sizes, with diameter in visual degrees, while the animal (monkey $\mathrm{AL}$ ) fixated at the white fixation spot. Sizes are not drawn to scale. Middle and bottom: Raster plots and peri stimulus time histogram (PSTH) of an example single unit activity aligned to stimulus onset for $0.3^{\circ}$ (left), $1^{\circ}$ (middle), and $4^{\circ}$ (right). Note the rhythmic activity specifically elicited by the $1^{\circ}$ stimulus.

(B) Multi-unit activity (MUA) response to each size, averaged across $\sim 40$ trials. The MUA was obtained from the same electrode from which the single unit activity in A was isolated. Similar to the single unit activity, MUA rhythmicity was elicited by the $1^{\circ}$ stimulus.

(C) Power spectra for every MUA trial (grey) shown in B. Power spectra were calculated from $200 \mathrm{~ms}-$ $1200 \mathrm{~ms}$ after stimulus onset. Black line shows the average power spectrum and red dots are the peak frequency of each trial's power spectrum.

(D) Normalized population power spectra of MUA for each size averaged across channels for monkey AL (left, $n=248$ channels) and monkey DP (right, $n=390$ channels). Theta frequency range is delineated by dashed lines. Error bars are \pm 1 standard error of the mean (SEM). 
(E) Normalized mean MUA (blue) and theta power (red) across different sizes, averaged across all channels for each monkey. Error bars are \pm 1 SEM.

(F) Median peak frequency of all channels at different sizes for each monkey. For every channel, we calculated the power spectrum and identified the frequency with the strongest power. Black dots are the medians of the distribution.

\section{Theta oscillations of MUA are modulated by stimulus size}

A fundamental aspect of V1 neuron responses is that activity strength increases with stimulus size, reflecting a spatial summation in the receptive field (RF). When stimulated beyond the summation field, V1 neuron responses tend to be suppressed ${ }^{42,43}$. To establish the link between this size tuning of V1 and theta oscillations, we first tested whether theta oscillations might be observed when we presented a static black stimulus of varying sizes at the RF location of V1 neurons. Figure 1 A top shows the activity of a V1 neuron, for which varying the stimulus size modulated the firing rate, both in the single-unit activity (SUA, Figure $1 \mathrm{~A}$ ) and in the multi-unit activity (MUA) (Figure $1 \mathrm{~B}$ ). Increasing the stimulus size from $0.3^{\circ}$ to $1^{\circ}$ increased the neuronal response but increasing the size to $4^{\circ}$ reduced it again. This observation reflects the known size tuning property of neurons in the visual cortex ${ }^{42,43}$. In addition to the difference in firing rate, this example demonstrates that theta oscillatory activity $(3-8 \mathrm{~Hz})$ emerged when the RF was presented with a specific size, $1^{\circ}$, near the peak of the size tuning function. The theta rhythmic activity can be seen in the PSTH of SUA and in the MUA time course. To quantify this oscillatory activity, we calculated the MUA power spectrum at the single-trial level from $200 \mathrm{~ms}$ to $1200 \mathrm{~ms}$ after stimulus onset (Figure $1 \mathrm{C}$ ). Compared to other stimulus sizes, the $1^{\circ}$ stimulus consistently induced theta rhythmic activity at individual trials, with higher theta band power and a more consistent peak frequency in the theta range.

At the population level, across recording locations, we found that theta oscillations were consistently modulated by stimulus size in both monkeys (Figure $1 \mathrm{D}$, left and right for different monkeys). There were no or only negligible theta oscillations in the pre-stimulus baseline period as well as for the smallest and biggest stimulus sizes. Instead, the power spectra consistently revealed the strongest theta peak for the medium stimulus sizes. To quantify this size dependent theta effect and compare it to classical size tuning estimates, we compared the mean MUA and theta power $(3-8 \mathrm{~Hz}$ ) across stimulus sizes (Figure $1 \mathrm{E}$ ). Both mean MUA and theta power were modulated by size ( $p<0.001$ for both monkeys, $n=$ 248 for monkey $A$ L and $n=390$ for monkey DP, Friedman test). Increasing stimulus size led to an increase in mean MUA and theta power up to a certain point where increasing the stimulus size further led to a decrease of both neural measures.

To get a more detailed view of theta oscillation frequency distribution at the population level, we identified the median peak frequency of every channel (Figure $1 \mathrm{C}$ ) and plotted the distribution of peak frequencies across recording channels as a function of stimulus size (Figure $1 \mathrm{~F}$ ). For some of the tested stimulus sizes, more than half of the channels showed a spectral peak at the theta frequency. At size $0.75^{\circ}$ where theta power was the highest, $60 \%$ of channels in monkey AL and $58 \%$ of channels in monkey DP were in the theta frequency. This means that the population results (Figure $1 \mathrm{D}$ and $\mathrm{E}$ ) were not dominated by only a few channels with particularly strong theta oscillations. In addition, this analysis revealed a trend of increasing peak frequency from the theta $(3-8 \mathrm{~Hz})$ to the alpha $(9-12)$ range with increasing size (significant difference between sizes, $p<0.001$ for both 
monkeys, $\mathrm{n}=248$ for monkey AL and $\mathrm{n}=390$ for monkey DP, Friedman test). Finally, the width of peak frequency distributions at sizes with strong theta, e.g., size $0.75^{\circ}$, was narrower than the distribution at sizes with weak theta, e.g., size $4^{\circ}$. The channels at $0.75^{\circ}$ were concentrated around theta frequency. At $4^{\circ}$, although there were channels with theta peak frequency, the concentration was less dense, and more channels were spread to higher frequencies. This means that MUA across channels was more likely to fluctuate consistently at a theta frequency as a response to some stimulus sizes compared to other sizes.

These results demonstrate that theta oscillations can be observed in V1. Different from previous studies in the visual cortex that reported theta oscillations when multiple stimuli ${ }^{32,32,34,37}$ are presented at the same time, we found that theta oscillations can be observed when the animal sees a single stimulus at the optimal size. Whereas theta power was strongest for intermediate stimulus sizes around $1 \mathrm{deg}$, following neuronal size tuning, peak frequency tended to increase with stimulus size towards the alpha range.

A

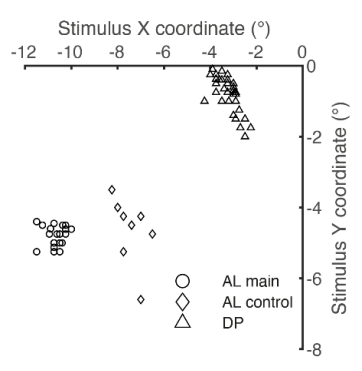

B

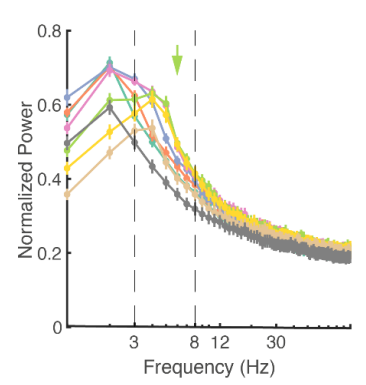

C

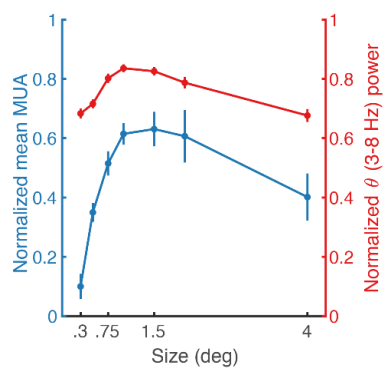

D

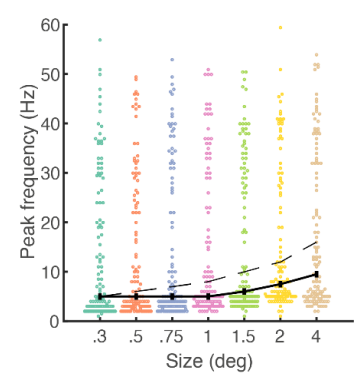

Figure 2. Frequency of theta oscillations shifted with different eccentricities

(A) Stimulus position coordinates of each session for the main sessions of monkey AL (circle), control sessions of monkey AL (diamond), and monkey DP (triangle)

(B) Normalized population power spectra of MUA for each size averaged across channels in the control sites in monkey AL. Theta frequency range is delineated by dashed lines. Green arrow denotes the peak frequency of size $1.5^{\circ}$ from the main sessions (Figure $1 \mathrm{D}$ left). Error bars are $\pm 1 \mathrm{SEM}$.

(C) Normalized mean MUA (blue) and theta power (red) across different sizes, averaged across all channels. Error bars are \pm 1 SEM.

(D) Median peak frequency of all channels at different sizes for each monkey. Black dots with the continuous line are the medians of the distribution. Dashed black line indicates the medians of the distributions from the main sessions for comparison(Figure $1 \mathrm{~F}$ left).

\section{Eccentricity as a factor influencing peak frequency}

When comparing the power spectra in Figure $1 \mathrm{D}$, we noticed a difference in the peak frequencies that were obtained from measurements in the two monkeys. Throughout, the spectral estimates obtained from monkey AL showed a higher peak frequency at 5 - 6 $\mathrm{Hz}$, than the ones in monkey DP at $4 \mathrm{~Hz}$. We reasoned that this difference in peak frequency might be explained by the difference in recording eccentricities of each monkey (Figure 2 A). To test for this possibility and to expand recording locations, we performed additional recordings from lower eccentricities in monkey AL (Figure $2 \mathrm{~A}$, diamond). 
In these less eccentric recording locations, we could again elicit theta oscillations (Figure $2 \mathrm{~B}$ ) and theta power varied according to stimulus size (Figure $2 \mathrm{C})(\mathrm{p}<0.001, \mathrm{n}=$ 120 , Friedman test). Theta peaks were most prominent at stimulus sizes $1.5^{\circ}, 2^{\circ}$, and $4^{\circ}$. Interestingly, recording from more central locations in the same animal shifted the peak of theta oscillations from $5-6 \mathrm{~Hz}$ to $4 \mathrm{~Hz}$ (compare the spectral peak of Figure $1 \mathrm{D}$ left to $2 \mathrm{~B}$ ). Thus, presenting the same stimulus size at a more central location in the visual field lowered the spectral peak to $4 \mathrm{~Hz}$.

Spectral peak distribution identified at the single trial level in these locations (Figure $2 \mathrm{D})$ also showed similar features as those in the more peripheral location (Figure $1 \mathrm{~F}$ ). Many channels displayed rhythmic neural activity concentrated at the theta frequency. However, the percentage of channels with theta frequency was lower in these locations. At size $2^{\circ}$, where the occurrence of theta channels was the highest, $53 \%$ of the recorded channels showed theta rhythmicity. This number was slightly lower than the percentage of theta channels in the more peripheral locations, $60 \%$. As before, there was also a trend of increasing peak frequency with increasing sizes (significant difference between sizes, $p<$ $0.001, n=120$, Friedman test), although this was more obvious in the larger stimulus sizes $\left(1.5^{\circ}-4^{\circ}\right)$. As Figure $2 \mathrm{D}$ shows, peak frequencies were lower in the more central locations than in the peripheral locations (statistically significant difference for size $0.75^{\circ}[p<0.001]$, $1^{\circ}[p<0.001], 1.5^{\circ}[p<0.001], 2^{\circ}[p<0.001]$, and $4^{\circ}[p=0.004]$, Mann-Whitney test). The rate of frequency increase with size was also lower in the more central locations (compare the solid and dashed black lines in Figure 2 D).

These results thus support and extend our observations for the size tuning of theta rhythmicity. We found that theta oscillations in V1 were not only affected by stimulus size, but also influenced by stimulus eccentricity. Comparing the mean MUA for two different eccentricities, our result is similar to what has been reported previously for an increase of receptive field size with eccentricity ${ }^{43}$. The peak of population size tuning slightly shifted to a larger stimulus size for more peripheral (Figure $1 \mathrm{E}$ left) compared to central stimulus locations (Figure $2 \mathrm{C}$ ). Furthermore, the observation of theta oscillations in multiple recording locations is indicative that these theta oscillations might contribute to the more general V1 function.

\section{Theta oscillations of MUA are modulated by stimulus contrast}

Having established the dependence of theta oscillation on stimulus size, we tested the dependence on a different stimulus property known to modulate V1 response, contrast. Responding to different contrast intensities is one of the fundamental functions of the visual system ${ }^{44}$. Investigating the relation of this function to the emergence of theta oscillations is therefore important. To test this, we stimulated the receptive field with a black disk with varying contrast levels relative to its background. The size of the black disk was optimized to elicit theta oscillations from a size-tuning test conducted beforehand. Figure $3 \mathrm{~A}$ shows an example channel MUA response to a stimulus with different contrast levels. A low contrast stimulus (Figure $3 \mathrm{~A}$ left) induced weak activity overall and no discernible theta oscillations (Figure $3 \mathrm{~B}$ left). When we increased the contrast (Figure $3 \mathrm{~A}$ mid), we could observe theta oscillations emerging at the single-trial level and on the average power spectrum (Figure $3 \mathrm{~B}$ mid). Increasing contrast further also increased the strength of theta oscillations (Figure $4 \mathrm{~A}$ and $4 \mathrm{~B}$ right). However, the increase of theta oscillation strength from mid to high contrast was not as pronounced as the increase in strength from low to mid contrast. 
The trend that theta oscillations increase with contrast can also be seen at the population level (Figure $4 \mathrm{C}$, significantly different power across contrasts at $p<0.001$ for both monkeys, $n=104$ for monkey $A L$ and $n=126$ for monkey DP, Friedman test). At low contrast, there was no distinctive theta peak in the power spectra. A theta peak became observable at mid contrast levels $(25-30 \%)$ and became more prominent at the highest tested contrast.

Similar to our observations in the size domain, many channels showed peak frequencies in the theta range (Figure $4 \mathrm{E}$ ). At $100 \%$ contrast stimulus, $76 \%$ of channels in monkey AL and $54 \%$ of channels in monkey DP displayed a peak frequency in the theta range. Although contrast overall had a statistically significant influence on peak frequency ( $p$ $<0.001, n=104$ for monkey $A L$ and $p=0.017, n=126$ for monkey DP, Friedman test), there was no obvious monotonic increase or decrease of peak frequency between contrasts. However, there was an increase of peak frequency from $25 \%$ to $100 \%$ contrast in monkey $\mathrm{AL}$ (Figure $3 \mathrm{E}$ top).

These results demonstrate that in addition to a stimulus' size, its contrast also affects theta oscillations. Theta oscillation power increased non-linearly as contrast increased. Different to what we found with stimulus size, where peak frequency increased with size, peak frequency did not follow an obvious trend across contrasts. 
A

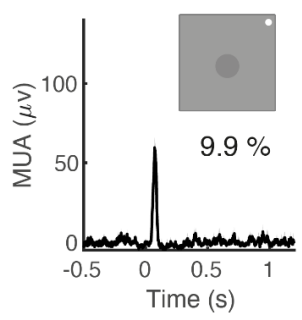

B

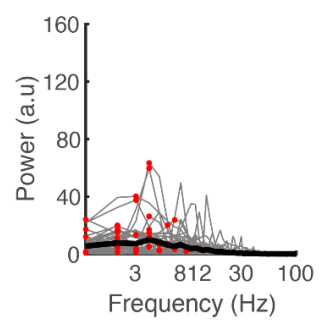

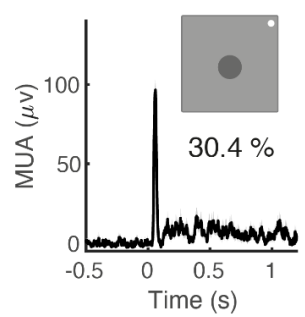

C
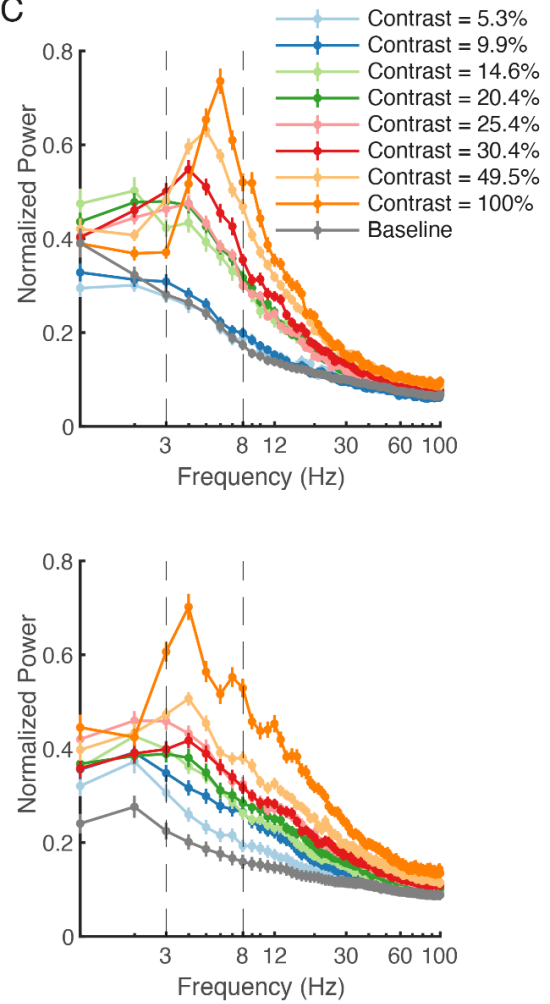

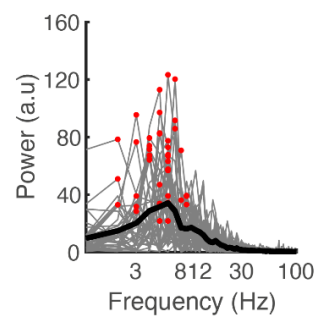

D

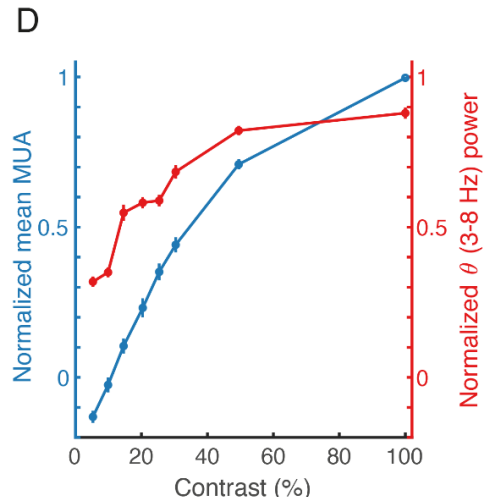

E
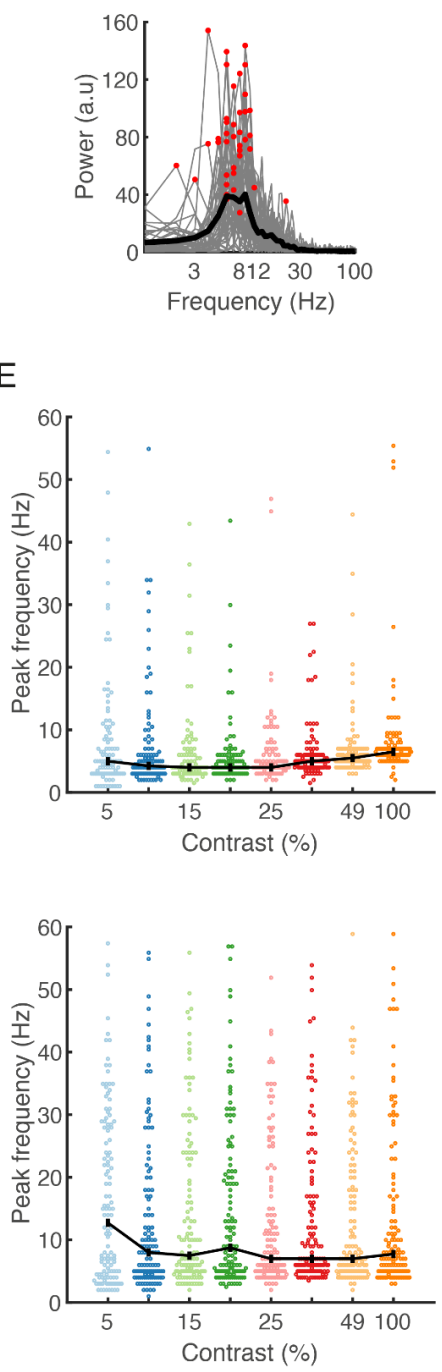

Figure 3. Theta oscillations at different stimulus contrasts

(A) Example MUA response to 3 different contrast levels, 9.9\%, 30.4\%, and $100 \%$. The size of the stimulus was optimized to elicit theta oscillations. The response was averaged across 40 trials. We can see theta oscillations in the MUA response to the $100 \%$ contrast stimulus.

(B) Power spectra for every MUA trial (grey) in A for each contrast level. Power spectra were calculated from $200 \mathrm{~ms}-1200 \mathrm{~ms}$ after stimulus onset. Black line shows the average power spectrum and red dots are the peak frequency of each trial's power spectrum. Theta oscillations increase with contrast.

(C) Normalized population power spectra of MUA for each contrast averaged across channels for monkey AL (top, $n=104$ channels) and monkey DP (bottom, $n=126$ channels). Theta frequency range was delineated by dashed lines. Error bars are \pm 1 SEM.

(D) Normalized mean MUA (blue) and theta power (red) across different contrasts, averaged across all channels for each monkey. Error bars are \pm 1 SEM. 
bioRxiv preprint doi: https://doi.org/10.1101/2021.11.30.470367; this version posted December $1,2021$. The copyright holder for this

preprint (which was not certified by peer review) is the author/funder, who has granted bioRxiv a license to display the preprint in perpetuity. It is made available under aCC-BY-NC-ND 4.0 International license.

(E) Median peak frequency of all channels at different contrasts for each monkey. Black dots are the medians of the distribution.

A

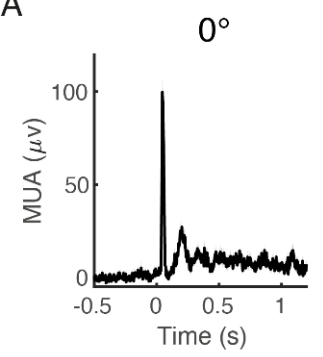

B

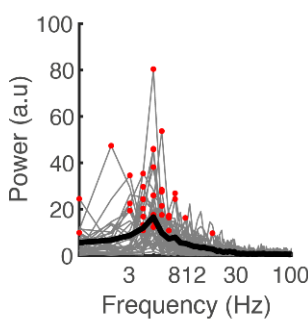

C
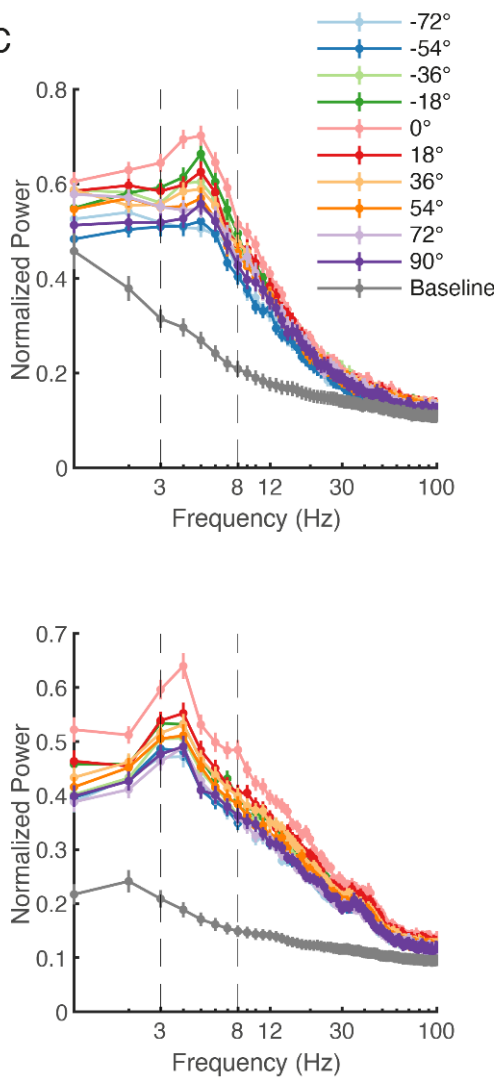

$54^{\circ}$
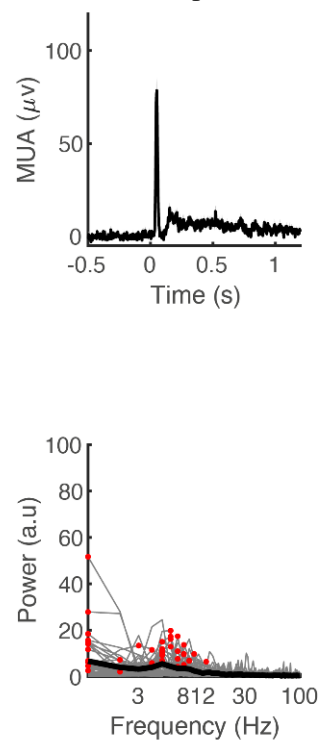

$108^{\circ}$
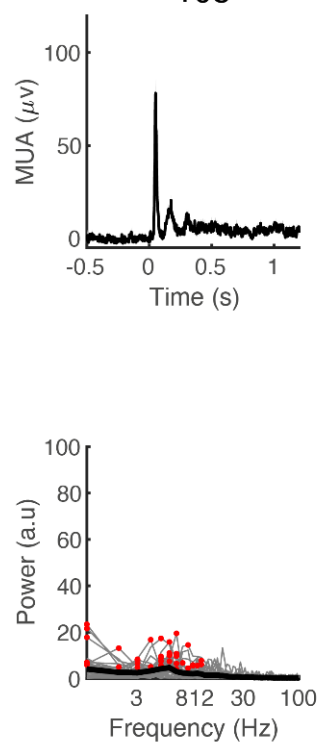

$162^{\circ}$
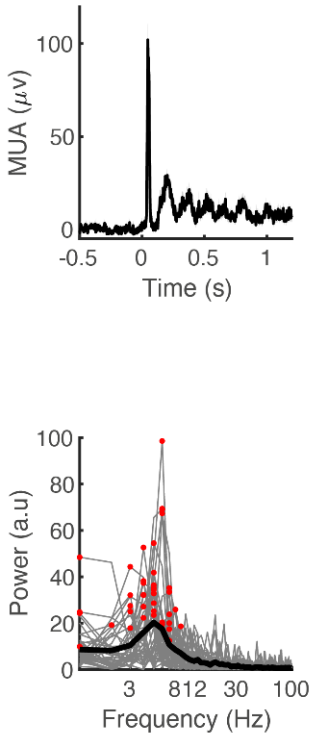

D

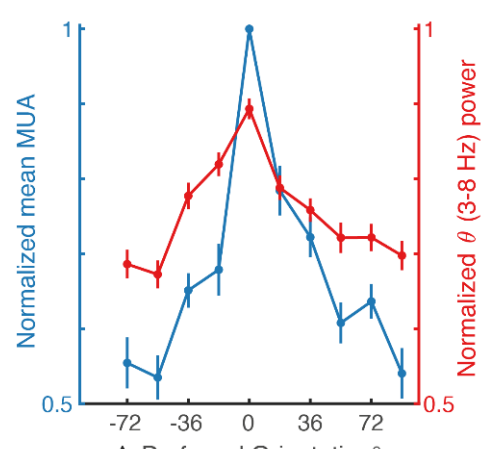

$\triangle$ Preferred Orientation ${ }^{\circ}$

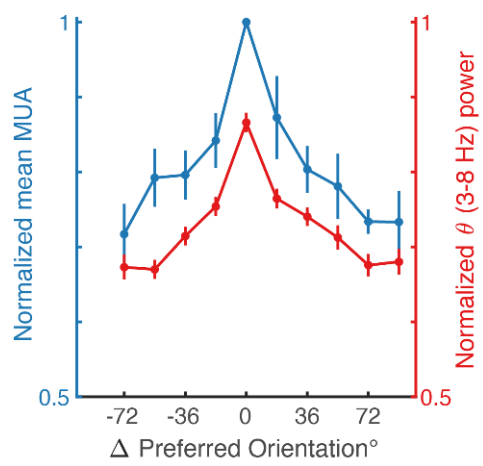

$E$
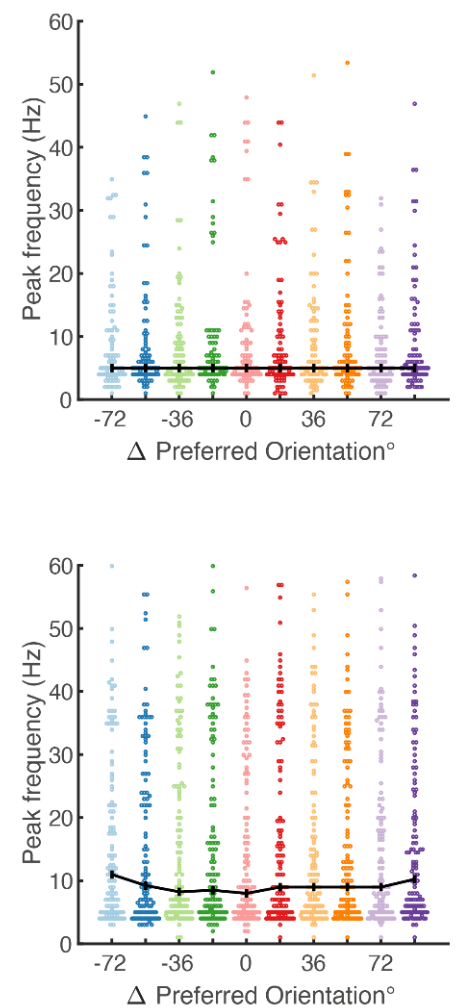

Figure 4. Theta oscillations at different stimulus orientations

(A) Example MUA response to 4 different grating orientations, $0^{\circ}, 54^{\circ}, 108^{\circ}$, and $162^{\circ} .0^{\circ}$ corresponds to vertical orientation and each positive orientation increment rotates the grating counterclockwise. The 
size of the grating was optimized to elicit theta oscillations. The response was averaged across $36-44$ trials. We can see theta oscillations most clearly in the MUA response to $162^{\circ}$ orientation.

(B) Power spectra for every MUA trial (grey) in A for each orientation. Power spectra were calculated from $200 \mathrm{~ms}-1200 \mathrm{~ms}$ after stimulus onset. Black line shows the average power spectrum and red dots are the peak frequency of each trial's power spectrum. The strongest theta oscillations can be seen for the $162^{\circ}$ orientation.

(C) Normalized population power spectra of MUA for each orientation averaged across channels for monkey AL (top, $n=94$ channels) and monkey DP (bottom, $n=126$ channels). Orientation preference was defined as the orientation that induced the highest mean MUA for each channel (orientation $0^{\circ}$ ). Other orientations were expressed as degree deviation from the preferred orientation. Theta frequency range was delineated by dashed lines. Error bars are \pm 1 SEM.

(D) Normalized mean MUA (blue) and theta power (red) across different orientations, averaged across all channels for each monkey. Error bars are \pm 1 SEM.

(E) Median peak frequency of all channels at different orientations for each monkey. Black dots are the medians of the distribution.

\section{Theta oscillations of MUA are modulated by stimulus orientation}

One of the most remarkable functions of $\mathrm{V} 1$ is the transformation of orientation insensitive visual information arising from lateral geniculate neurons to orientation-selective information as measured in V1 neurons ${ }^{45}$. After we established the dependence of theta oscillations to size and contrast, we, therefore, investigated the relation of theta oscillations to orientation processing in V1.

We presented a static full contrast sinusoidal grating with different orientations in the RF. Figure 4 A shows an example MUA response to four different grating orientations. As expected, MUA was modulated by orientation. In this example, the neuronal population preferred orientations close to vertical, $162^{\circ}$ and $0^{\circ}$ (Figure $4 \mathrm{~A}$ right). Orientations that are perpendicular to the preferred horizontal orientation, $54^{\circ}$ and $108^{\circ}$ (Figure $4 \mathrm{~A}$ middle), induced weaker overall responses. Interestingly, we also observed that theta oscillations were similarly affected by grating orientation (Figure $4 \mathrm{~B}$ ). Theta oscillations were strong at the preferred orientations, $162^{\circ}$ (Figure $4 \mathrm{~B}$ right), and $0^{\circ}$ (Figure $4 \mathrm{~B}$ left), but weak at orientations perpendicular to $162^{\circ}$ (Figure $4 \mathrm{~B}$ middle).

At the population level, we determined channel-averaged power spectra based on the preferred orientation of each channel (Figure $4 \mathrm{C}$ ). Preferred orientation was defined as the orientation which elicited the highest mean MUA. The results show that theta oscillations are affected by orientation ( $p<0.001$ for both monkeys, $n=94$ for monkey AL and $\mathrm{n}=126$ for monkey DP, Friedman test). In both monkeys, the preferred orientation induced the strongest theta oscillations across recording channels. The further an orientation deviated from the preferred one, the weaker the theta oscillations (Figure 4 D).

Similar to the other tested stimulus properties, the peak frequency for the majority of channels again clustered at the theta range, which was true for all orientations (Figure 4 E). Around half of the channels showed peaks in the theta range for monkey AL ( $46-53 \%$ ) and monkey DP (41 - $48 \%$ ). There was no obvious peak frequency difference between different orientations (non-significant difference between orientations, $p=0.133, n=94$ for monkey $A L$ and $p=0603, n=126$ for monkey DP, Friedman test).

These results show that orientation, one of the best described stimulus properties of V1, also affects theta oscillations. Theta power is the highest at the preferred orientation and gradually becomes weaker as orientation moves further from the preferred one(s). It is 
bioRxiv preprint doi: https://doi.org/10.1101/2021.11.30.470367; this version posted December $1,2021$. The copyright holder for this preprint (which was not certified by peer review) is the author/funder, who has granted bioRxiv a license to display the preprint in perpetuity. It is made available under aCC-BY-NC-ND 4.0 International license.

interesting to note that after we showed theta oscillations with a uniform black circular stimulus, we also observed theta oscillations with a new stimulus, a sinusoidal grating. This finding might therefore argue against theta being exclusively produced by a specific set of stimuli (e.g. a black circle).

A

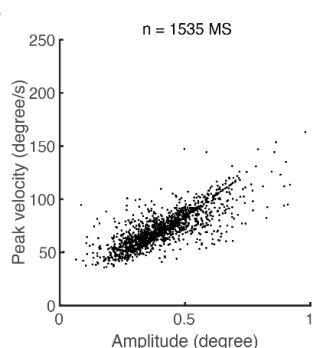

C

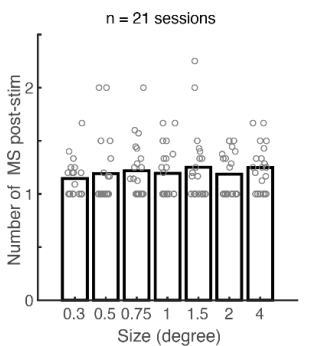

E

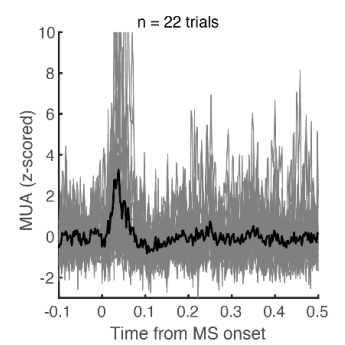

Monkey AL

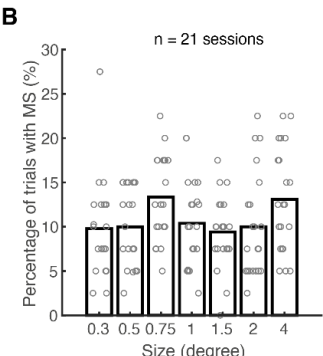

D
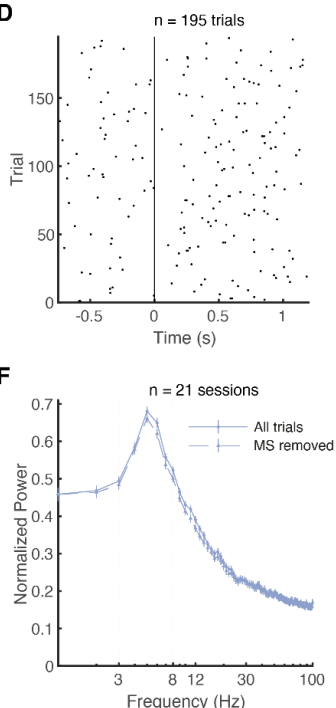

G
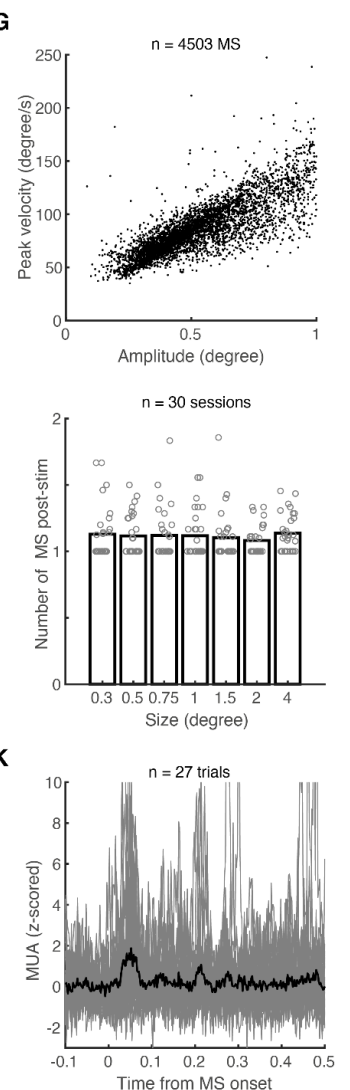

Monkey DP

H
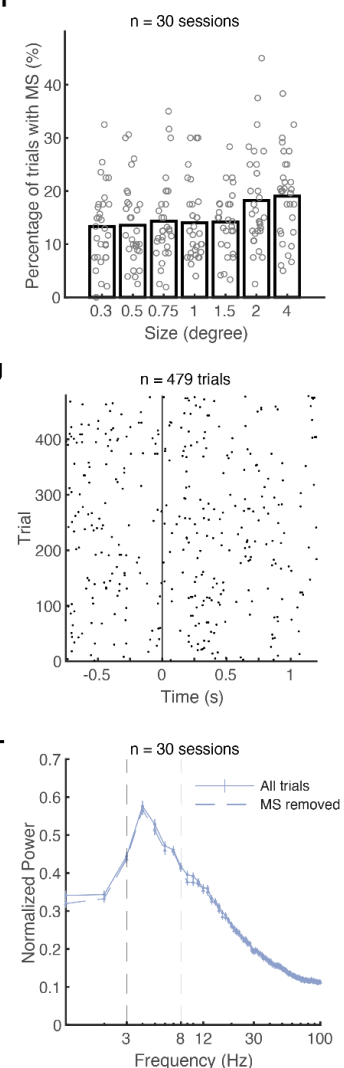

Figure 5 Theta oscillations do not depend on microsaccades

(A) Microsaccades main sequence ( $n=1535$ from 21 sessions), showing a positive relationship between microsaccade amplitude and peak velocity.

(B) Mean percentage of trials with microsaccades across stimulus sizes. Each dot represents a single session. Microsaccades were rare events. On average, microsaccades occurred on $\sim 5 \%$ of trials across sizes.

(C) Number of microsaccades in the post-stimulus period in trials where microsaccades occurred after stimulus onset. Each dot represents a single session. On average, microsaccades once during the whole post-stimulus period.

(D) Microsaccade raster plot for size $0.75^{\circ}$ combined across sessions. Each dot shows the onset time of microsaccades during a particular trial. Vertical line delineate stimulus onset. There is no obvious rhythmicity in the microsaccades timing across trials.

(E) Example microsaccade-triggered MUA from one session, combined across stimulus sizes. Grey lines show individual trials $(n=15)$ MUA. Microsaccades induced a brief increase of MUA which quickly ( $100 \mathrm{~ms}$ ) returned to pre-microsaccade level. There is no obvious rhythmicity in the MUA after microsaccades onset.

(F) Power spectra of stimulus size $0.75^{\circ}$ before (solid line) and after removing trials with microsaccades in the post-stimulus period.

(G-L) Same plots but for the second monkey. 


\section{Theta rhythmic activation of $\mathrm{V} 1$ unlikely related to microsaccades}

Having established the stimulus dependence of V1 theta oscillations, we wondered whether this rhythmic activity might be related to behaviour. Previous research has established an association between neural theta oscillations and microsaccade ${ }^{5,6}$ which occur every $\sim 250 \mathrm{~ms}^{4-6}$. As the activity of $\mathrm{V} 1$ neurons is modulated by microsaccades ${ }^{46-}$ 48 , it seemed possible that theta oscillations in V1 might be caused by rhythmic alterations of neural activity in response to the occurrence of microsaccades or similarly reflect the occurrence of theta-rhythmic microsaccades. To test this possibility, we extracted microsaccades recorded during the size tuning task with a widely used algorithm ${ }^{49}$. The positive relationship between microsaccade amplitude and peak velocity confirmed the validity of our microsaccade extraction (Figure $4 \mathrm{~A}$ and $\mathrm{G}$ for each monkey).

During our stimulation paradigm, microsaccades were not present in every trial. After stimulus onset and across sizes, microsaccades occurred in around $10 \%$ of the trials for monkey $\mathrm{AL}$ and $15 \%$ of the trials for monkey DP (Figure $5 \mathrm{~B}$ and $\mathrm{H}$ ). This microsaccades occurrence pattern is substantially different from our observations that post-stimulus neural theta oscillations are consistently present across trials (Figure $1 \mathrm{C}$ middle as an example). However, the percentage of trials with post-stimulus microsaccades are slightly different across sizes $(p=0.04, n=21$ for monkey AL and $p=$ $0.008, \mathrm{n}=30$ for monkey DP, Friedman test). In monkey AL (Figure $5 \mathrm{~B}$ ), the stimulus size with the highest percentage of microsaccades is $0.75^{\circ}$, a size that induced the strongest theta power (Figure $1 \mathrm{D}$ left). In monkey DP, microsaccades most often occurred with the largest stimulus size (Figure $5 \mathrm{H}$ ), which in turn did not induce theta oscillations (Figure $1 \mathrm{D}$ right).

In trials where microsaccades occurred after stimulus onset, microsaccades typically occurred only once (Figure $5 \mathrm{C}$ and I). Furthermore, the number of microsaccades during the post-stimulus period was not different across stimulus sizes (Figure $5 \mathrm{C}$ and $\mathrm{I}$ ) ( $\mathrm{p}>$ 0.05 , Friedman test). Even at a stimulus size that induced strong neural theta oscillations in both monkeys, $0.75^{\circ}$ (Figure $5 \mathrm{D}$ and $\mathrm{J}$ ), no theta rhythmic occurrence of microsaccades could be seen. Therefore, these observations are inconsistent with the hypothesis that theta oscillations are systematically caused by a theta-burst of microsaccades causing neuronal theta oscillations.

At the neural level, our observations are consistent with previous reports e.g. 48 : microsaccades evoked a temporary increase in MUA which peaked around $50 \mathrm{~ms}$ after microsaccade onset and quickly returned to baseline levels (Figure $5 \mathrm{E}$ and $\mathrm{K}$ ). This transient change of MUA is too brief to account for one cycle of theta oscillations (125 $333 \mathrm{~ms}$ ) and did not display any rhythmicity after the initial transient.

In order to further investigate the relationship between microsaccades and theta oscillations, we removed all trials that contained microsaccades and analysed them with regard to the presence of theta oscillations. If microsaccades are the generator of neural theta oscillations, we expect their disappearance after the removal of microsaccades. We found that theta oscillations were still present after removing trials with microsaccades (Figure $5 \mathrm{~F}$ and $\mathrm{L}$ ). Although the power in the neural theta band (averaged across $3-8 \mathrm{~Hz}$ ) was slightly, but significantly, lower after the removal of trials with microsaccades ( $p<0.001$ for both monkeys, Wilcoxon signed-rank test), this decrease was only $2-3 \%$ for both monkeys. These observations indicate that 
microsaccades are not the origin of theta rhythmicity in V1 neurons, although microsaccades might further amplify a stimulus-induced neural theta rhythm.

Taken together, the above findings show that microsaccades cannot explain the emergence of theta oscillations in V1 under our task conditions. In our preparation, microsaccades were rare, did not occur rhythmically, and theta oscillations were still present in the MUA during trials without microsaccades. Note that this does not mean that theta oscillations and microsaccades are completely independent. The reverse influence direction, that theta oscillations are involved in the generation of microsaccades, could still be true.

A

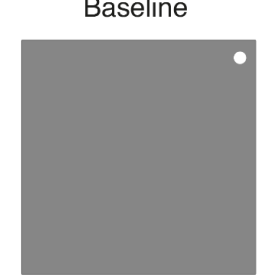

$1000 \mathrm{~ms}$
Stimulus on

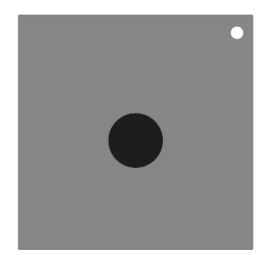

$500-1500 \mathrm{~ms}$
Target on

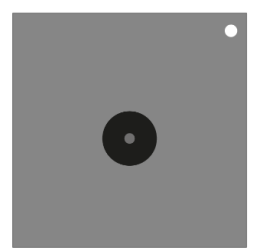

$1000 \mathrm{~ms}$

Monkey AL

B

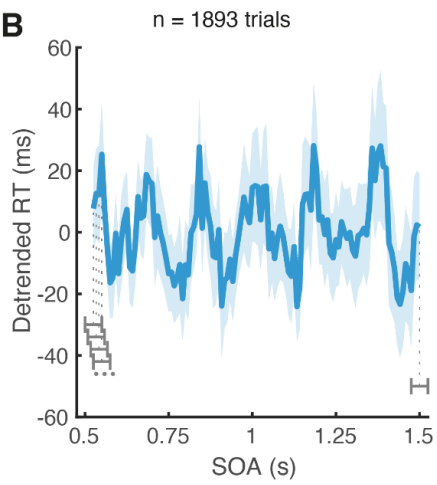

C

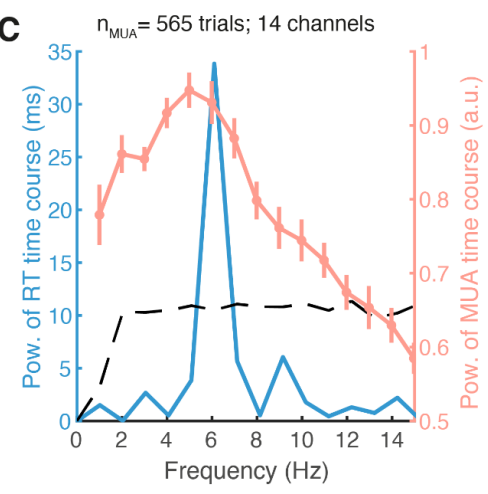

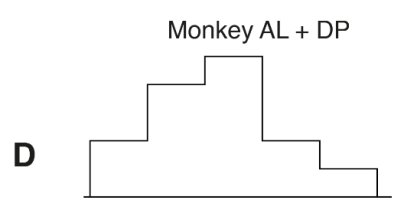

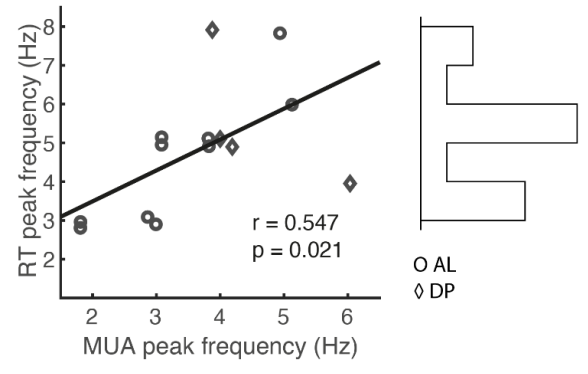

Figure 6 Theta oscillations in multi-unit activity and behaviour

(A) Behavioural task. The monkeys had to detect a small luminance change (grey dot), in the centre of a stimulus (black disk) and make a saccade toward the stimulus. The interval between stimulus and luminance change, stimulus onset asynchrony (SOA), was varied densely between 500 to $1500 \mathrm{~ms}$.

(B) Left: reaction times (RT) as a function of SOA from one session. Grey horizontal bars illustrate the binning procedure to obtain RT at a particular SOA. Note the rhythmicity in the time course of the RT.

(C) Power spectrum of the time course of the RT in B (blue), superimposed with the average neural power spectra recorded during the task (orange). Black dashed line is the threshold of statistical significance for the RT power spectrum after correction for multiple comparisons $(\langle=0.05 / 15$ frequencies $=0.0033$ ).

(D) Scatter plot showing the relationship between peak frequency of MUA power spectra and peak frequency of RT power spectra ( $n=14$ sessions). Sessions are combined for both monkeys. We introduced a small jittering to the data points in the scatter plot for illustration purposes only to improve visibility because of the overlaps of some data points. The correlation coefficient and regression line were calculated from the non-jittered data. There was a significant positive 
relationship between MUA peak frequency and RT peak frequency. Marginal histograms showed the concentration of peak frequency of both MUA (top histogram) and RT (bottom histogram) in the theta range.

\section{MUA and behaviour are modulated by a similar rhythm}

An increasing number of studies in humans e.g. 8,9 and macaques ${ }^{24,34}$ shows rhythmicity in vision-guided performance measures collected from attention tasks involving multiple visual stimuli and which has been interpreted as evidence for a rhythmic sampling of visual stimuli. For the present study, we wondered therefore whether the stimulusinduced V1 theta oscillations might be similarly related to a behavioural rhythm.

To this end, we trained the monkeys to perform a simple visual detection task (Figure $6 \mathrm{~A}$ ). The monkeys first had to view a central fixation spot while a visual stimulus was presented that induced theta oscillations in the simultaneously recorded V1 MUA. The monkeys had to covertly attend to this theta-inducing visual stimulus to detect a small luminance change in its centre. Detection of the luminance change was reported by making a saccade towards the location of change. The timing of the change varied on a trial-by-trial basis between 500 and 1500 ms following stimulus onset. Across trials, this experimental manipulation allowed us to systematically assess reaction times (RT) at different epochs of the stimulus-induced neural theta rhythm ${ }^{8,9}$. Our rationale was that if the stimulus elicits neural theta oscillations that are behaviourally relevant, presenting the luminance change at different times after stimulus onset should reveal a similar oscillatory structure in behavioural RT measures.

Indeed, plotting RTs from one experimental session as a function of visual target onset times revealed a regularly alternating pattern of shorter and longer RTs (Figure 6 B). Spectral analysis of this RT time course showed that RTs fluctuated at a theta frequency of 6 $\mathrm{Hz}$ (Figure $6 \mathrm{D}$, blue). In addition, we found that MUA oscillated at theta frequency (Figure 6 $B)$. The frequency of the neural oscillations peaked at $5 \mathrm{~Hz}$, closely resembling the frequency of behavioural oscillations $(6 \mathrm{~Hz})$. Across sessions in both monkeys, we found a positive moderate correlation between peak of MUA power spectra and peak of RT power spectra (Figure $5 \mathrm{C}$ ) ( $r=0.547$ [95 \% Cl $0.118-1$ ], $p=0.021$ one-tailed, $n=14$, Pearson correlation). Therefore, across sessions, MUA and RT tended to fluctuate at similar frequencies and it seems that the stimulus-induced theta rhythm in V1 translated into similar behavioural performance fluctuations.

\section{Discussion}

We found a stimulus-induced theta rhythmic neural activity in primate V1 which depended on basic stimulus properties such as size, contrast and orientation. The rhythms were not eliminated after excluding trials with microsaccades from the analysis. When monkeys actively performed a visual detection task, both MUA and RT measures were modulated at similar theta frequencies. In what follows, we discuss our findings in the context of previous reports of theta oscillations in the visual cortex, including what is known about the stimulus dependence of neural oscillations, and their relationship to visual perception. 
Theta oscillations in visual cortex

Neural theta oscillations have been observed in the temporal cortex $29,32,50, \mathrm{~V} 4$ $33,34,51$, and $\mathrm{V} 1{ }^{37,38}$. These studies collectively show that theta oscillations might occur during a wide set of cognitive task conditions in the context of attention ${ }^{37}$, visual working memory ${ }^{33}$, visual discrimination ${ }^{29}$, illusory contour ${ }^{51}$, binocular rivalry ${ }^{50}$, and passive fixation to multiple nearby stimuli ${ }^{32,34,38}$. The viewing of multiple stimuli was demonstrated to be an important factor in the emergence of theta oscillations in the mid- to higher visual areas. More specifically, theta oscillations seem to be produced by competition between populations of neurons representing different stimuli in IT ${ }^{32}$ and RF centre-surround competition in $\mathrm{V} 4{ }^{34}$. Stimulation of RF centre or surround alone did not induce theta oscillations in the recorded cortical areas. Instead, a balance between RF excitation and inhibition was necessary for the emergence of theta oscillations in the visual association cortex.

In V1, there are two studies reporting stimulus related theta oscillations. The first one measured theta oscillations in local field potentials (LFP) while monkeys performed an attention task ${ }^{37}$ involving multiple visual objects. This study reported that theta oscillations were reduced by selective visual attention. The second study reported theta oscillations in MUA while monkeys viewed an illusory contour induced from the presence of multiple illusion-inducing stimuli centred around $\mathrm{V} 1 \mathrm{RFs}^{38}$.

Our study extends these earlier observations and adds that the emergence of theta oscillations in V1 depends on basic visual stimulus properties. Similar to previous studies in the primate visual cortex ${ }^{32,34}$, our findings highlight the importance of basic neural computations at the RF level for the emergence of theta rhythmic neural activity.

Stimulus dependence and the emergence of theta oscillations in V1

Across all tested stimulus properties - size, contrast, and orientation - we noticed a pattern that theta oscillations tend to depend on spike rate. Stimulus parameters that were effective in driving the neural population also tended to be the ones effective in inducing theta oscillations. This finding points to the possibility that in V1, theta oscillations are inherent in the spiking pattern of the neural population. When a neural population is minimally activated, such as during the pre-stimulus period or during the presentation of non-optimal stimuli, theta oscillations are not likely to emerge or only emerge with low power. In contrast, an optimal visual stimulus strongly drives the theta oscillations.

A possible link can be made between theta oscillations and the preference of many V1 neurons to a stimulus drifting in theta frequency ${ }^{40,41,52,53}$. This finding is consistent with what was found with human functional magnetic resonance imaging (fMRI) ${ }^{54-57}$ and MEG 58,59 . This preference may indicate that many V1 neurons act as temporal filters which are tuned to theta frequencies. Therefore, if a static stimulus is presented to the neurons for a sustained period - the presentation condition in our study - the neurons will fire rhythmically at theta frequency. The more strongly a stimulus activates a neuron, the stronger the resulting theta activity is, consistent with what we found across stimulus properties. The possible inherent theta rhythmicity in many $\mathrm{V} 1$ neurons may be revealed only by a sufficiently efficient stimulus drive.

In addition to the effect of stimulus properties on theta power, we also found that theta peak frequency increased with both stimulus size and eccentricity. It is interesting to 
note that the opposite effect has been found for gamma oscillations in the LFP ${ }^{60-63}$ and MEG ${ }^{64}$. Gamma oscillation frequency has been observed to decrease with increasing stimulus size ${ }^{60,63}$ and eccentricity ${ }^{61,64}$, whereas the theta frequency increased under our conditions. As there is indicative evidence from human $\mathrm{fMRI}$ studies that the preferred temporal frequency of $\mathrm{V} 1$ neurons might increase from parafoveal to peripheral locations in V1 ${ }^{56,57}$, this effect might underlie the eccentricity dependent modulation of V1 theta frequencies. From these observations, a picture emerges that places the inherent filter properties of $\mathrm{V} 1$ neurons at the core for the emergence of theta-rhythmic neural activity in the primary visual cortex.

\section{Theta oscillations and microsaccades}

What might be the perceptual consequences of neural theta rhythms in the visual cortex? Previous studies showed a phase-locking between microsaccades and theta activity in V1 5,6. A similar relationship could not be observed in our study. First, we found that microsaccades did not occur in every trial; they were relatively uncommon events that occurred only in $10-15 \%$ of the trials. In contrast, theta oscillations were consistently present across trials. Second, when microsaccades occurred in the stimulus presentation period, they only occurred once per second on average, in agreement with previous reports 65,66. A possible explanation why we didn't find a rhythmic occurrence of microsaccades might be because of our shorter stimulus presentation period of $1.2 \mathrm{~s}$, compared to previous studies where the stimulus presentation could last for several seconds ${ }^{5,6}$. As microsaccades rate increases with longer fixation time ${ }^{4,67}$, it is therefore possible that microsaccades are more likely to become rhythmic during more prolonged fixation. Third, as a test for the dependence of theta oscillations on microsaccades, removing trials with microsaccades didn't eliminate neural theta oscillations, consistent with previous reports 34,37 . These findings together argue against the involvement of microsaccades in the generation of neural theta oscillations in our study.

Theta oscillations and rhythmic sampling

Multiple studies found rhythmic fluctuations of behavioural performance at theta frequency across different visual tasks $8,11-13,20,68,69$. These findings have been interpreted as a rhythmic sampling of the visual environment. It is interesting to note that most of the evidence for rhythmic sampling is found when observers are presented with multiple objects at the same time. For example, the study by Landau and Fries ${ }^{8}$ first presented the observers with a non-informative spatial cue. The observers then needed to attend to two stimuli because the target could appear in either. Therefore, rhythmicity in behavioural performance is often interpreted as attentional switching between multiple objects.

Here we provide one of few demonstrations of behavioural rhythmicity in the presence of one object, consistent with a recent behavioural study ${ }^{13}$. When monkeys had to detect a target inside a stimulus that was favourable for the emergence of theta, RT fluctuated at theta frequency. Our investigation and the recent behavioural study ${ }^{13}$ in humans provide evidence that behavioural rhythmicity does not always arise from competition, exploration, or attentional switching process between multiple stimuli ${ }^{28}$. These findings instead indicate rhythmic processing of a feature or a single location in space by the visual cortex neurons, which appears to be stimulus-driven ${ }^{32,34}$. 
Most evidence on the neural correlates of the behavioural rhythmicity points to the frontoparietal network $23,24,70-72$. This higher-level correlate of behavioural rhythmicity is indicative of a top-down attentional control of sampling ${ }^{73,74}$. Here, we show an additional sensory-driven correlate of behavioural rhythmicity as early as in V1, at the entrance point of visual cortical processing. The theta rhythm in V1 induced by a stimulus may bias further downstream processing of that stimulus, making the perception of it fluctuate rhythmically at a similar frequency. Our results thus agree with and extend previous human studies that showed a rhythmic involvement of early visual areas during visual search using a perturbation technique, transcranial magnetic stimulation (TMS) ${ }^{20,72}$.

The existence of theta across brain regions suggests multiple mechanisms and functions. It is possible that theta oscillations in early visual areas, such as V1 and V4 ${ }^{34}$, are involved when processing happens locally, such as in the case of viewing one stimulus or two nearby stimuli. This type of processing is well served by the small size of RFs in early visual areas. When a task requires attending to multiple locations or objects over a large area, which is a common testing condition of rhythmic behaviour in humans $8,11,20,68-70,75$ and monkeys $23-25,70,71$, top-down control regions are involved so the postulated attention spotlight ${ }^{76}$ can scan the visual field to process all stimuli ${ }^{77}$. In fact, theta oscillations in V4 are reduced when two stimuli are presented in different hemifields compared to when the two stimuli are presented close to each other ${ }^{34}$, consistent with the idea that theta oscillations in mid- and early visual areas are involved in local processing.

In summary, we provide evidence for the emergence of theta rhythmic neuronal activity in the primate primary visual cortex that is highly stimulus-dependent and appears to influence the dynamics of visual perception. This theta-rhythmic activity seems to directly arise from V1 filter properties and is indicative of a sensory-based sampling mechanism that operates in parallel to other rhythmic cognitive processes.

\section{Methods}

\section{Subjects}

Two healthy adult female rhesus monkeys (Macaca mulatta, monkey AL: age 5 years old and weight $8 \mathrm{~kg}$; monkey DP: age 6 years old and weight $9 \mathrm{~kg}$ ) participated in this study. We implanted a head post and a recording chamber over area V1 in the right hemisphere. Anaesthesia procedure, surgical procedure, implant methods, and postoperative conditions are described in a previous publication (Ortiz-Rios et al 2018). During the testing period, the monkeys were put under a fluid control procedure which did not impair the animals' physiology and welfare ${ }^{78}$. All procedures complied with UK Animals Scientific Procedures Act 1986 and European Council Directive 2010/63/EU.

\section{Neurophysiological recordings}

Neurophysiological data were collected by two types of electrodes used in different experimental sessions: (1) single tungsten electrodes with epoxylite coating (FHC, Bowdoin, USA) and (2) silicone linear probes with 16 (1 shaft) or 32 channels ( 2 shafts with $200 \mu \mathrm{m}$ spacing between the shafts), $150 \mu \mathrm{m}$ inter-electrode spacing, with platinum contacts (Atlas 
Neuroengineering, Leuven, Belgium). Single FHC electrodes were referenced to the stainless-steel guide tube used to penetrate the dura, while Atlas linear probes were referenced to a silver wire placed on the dura while the chamber was filled with saline. Electrodes were inserted daily into the right V1 of each monkey with a hydraulic micromanipulator (Narishige, Japan). Raw data were recorded at a sampling rate of $30 \mathrm{kHz}$ using a Blackrock Microsystems Cerebus system (Blackrock Microsystems, Utah, USA). Neurophysiological data were analysed using MATLAB-based NPMK software (Blackrock), custom-written MATLAB codes (Mathworks), and the FieldTrip Matlab software toolbox ${ }^{79}$. The envelope of multi-unit activity (MUA) ${ }^{80}$ was obtained by high-pass filtering (8th-order Chebyshev filter with $300 \mathrm{~Hz}$ cutoff frequency) the raw data, rectifying (taking the absolute values), and downsampling to $500 \mathrm{~Hz}$.

\section{Visual stimulation}

Stimulus presentation and monkey behaviour were controlled by MWorks (https://mworks.github.io). During the experiments, eye movements were tracked monocularly and recorded using an infrared-based eye-tracking system with $500 \mathrm{~Hz}$ sampling rate (EyeLink 1000, SR Research, Ottawa, Canada). Stimuli were presented with a ViewPixx LCD monitor with a $120 \mathrm{~Hz}$ refresh rate, $1920 \times 1080$ pixels resolution, and 24-inch diagonal display size (VPixx technologies, Saint-Bruno, Canada). The monitor luminance output was linearized by measuring the luminance of red, green, and blue using a photometer at 8 brightness levels repeated 10 times each. We fitted the luminance profile with a power function and applied the inverse of the power function during stimulus presentation. The viewing distance was $85 \mathrm{~cm}$.

RF location was estimated by presenting a black square (luminance $0.1 \mathrm{~cd} / \mathrm{m} 2$ ) in quick succession, $100 \mathrm{~ms}$ each, at various spots on the screen with a grey background (luminance $45 \mathrm{~cd} / \mathrm{m} 2)$. The animals fixated a white fixation dot $\left(0.3^{\circ}\right.$ diameter, $92 \mathrm{~cd} / \mathrm{m} 2$ luminance). Usually, we started by presenting a $1^{\circ}$ wide square in a non-overlapping 5 by 5 grid to find the rough location of the RF. The MUA responses to the RF stimuli were analyzed to find the spot where activation was highest. We considered this spot to be the centre of the RF location. Once we confirmed the rough RF location, the procedure was repeated with $0.5^{\circ}$ and $0.25^{\circ}$ squares to further delineate the RF location at a higher spatial resolution. The RF centre location obtained with $0.25^{\circ}$ stimulation was recorded and subsequent visual stimulation was always placed at this location. In the case where we recorded with multiple electrodes, we usually focused on the RF location of one or two channels with the strongest activation as judged by listening to the auditory-converted neural activity and/or by visual inspection of the MUA data from RF analysis.

For the passive viewing task, the animals were required to maintain fixation on a small white dot while various stimuli were presented at the receptive field (RF). All stimuli were always presented with a gray background ( $R G B[0.5,0.5,0.5]$ luminance $45 \mathrm{~cd} / \mathrm{m} 2$ ). Trials were initiated by the animals looking at the fixation dot followed by a fixation time of at least $1000 \mathrm{~ms}$. After that, the stimuli were presented for $1200 \mathrm{~ms}$. Animals were rewarded if they maintained fixation for the whole duration of the trial. If the animals moved their eyes outside the fixation window any time before the stimulus disappeared, the trials were aborted, and they weren't rewarded. The stimulation conditions from failed trials were included again randomly in the protocol to ensure adequate testing across the 
different task conditions. The radius of the fixation window was typically $1^{\circ}$. Depending on the animals' condition and motivation, the radius could be smaller, but never exceeded $1^{\circ}$.

To test the effect of stimulus size on theta oscillations, we presented a black (RGB $[0,0,0]$ luminance $0.1 \mathrm{~cd} / \mathrm{m} 2)$ disk with one of 7 possible sizes $\left(0.3^{\circ}, 0.5^{\circ}, 0.75^{\circ}, 1^{\circ}, 1.5^{\circ}, 2^{\circ}\right.$, and $4^{\circ}$ diameter). The size of the stimulus varied on a trial by trial basis. For most of the recordings, we presented each size for 40 trials; however, in some sessions, we presented more trials per size depending on the animals' motivation. After the size tuning recording was finished, the data was analyzed to identify the size which induced the strongest theta power. This size was recorded and used to present stimuli for the contrast and orientation experiment.

To test the effect of contrast, we presented a grey disk with varying contrast relative to the grey background. On a single trial, we presented the disk with 8 possible contrasts (5.3\%, $9.9 \%, 14.6 \%, 20.4 \%, 25.4 \%, 30.4 \%, 49.5 \%, 100)$. The contrast was calculated as Michelson contrast and varied every trial. For most recordings, we presented each contrast for at least 40 trials. For one session in monkey DP, we only presented only 20 trials for each contrast. In some sessions, depending on the motivation of the monkey, we presented more than 40 trials per contrast.

To test the effect of orientation, we presented a sinusoidal grating with a circular envelope with varying orientations. On a single trial, we presented the grating at one of 10 possible orientations. The tested orientations were linearly spaced from $0^{\circ}$ (vertical orientation) to $162^{\circ}$ with $18^{\circ}$ increment. The grating was presented at full contrast. Spatial frequency was 1 cycle per degree for monkey AL and 2 or 3 cycles per degree for monkey DP. For most recording sessions, each orientation was presented for 30 - 40 trials.

For the detection task, the monkeys maintained fixation for $1000 \mathrm{~ms}$ before a stimulus was presented in the RF. The stimulus was a black disk (luminance $0.1 \mathrm{~cd} / \mathrm{m} 2$ ) on a grey background (luminance $45 \mathrm{~cd} / \mathrm{m} 2$ ). The stimulus size was optimized to generate theta oscillations from a size tuning passive viewing task performed before the detection task. The stimulus size that induced the strongest theta oscillations was used as the stimulus size in the detection task. After a random 500 to 1500 ms of stimulus presentation, a target appeared in the centre of the stimulus and stayed on the screen for a maximum of $1000 \mathrm{~ms}$. The monkeys had to detect the target by making a saccade toward the stimulus within 1000 ms after target onset. A successful saccade to the stimulus was rewarded. Fixations break from the fixation point at any time before target onset would abort the trial. In $25 \%$ of trials, we presented catch trials where there was no target presentation. In the catch trials, the monkeys were rewarded for maintaining fixation for $1500 \mathrm{~ms}$.

\section{Data analysis}

\section{Passive viewing task}

Our main unit of analysis is the MUA collected per electrode for the FHC electrodes and per electrode channel for the linear probes. For our main analysis, we only included visually responsive channels. To determine the visually responsive channels, we compared the 100 ms post-stimulus onset MUA to the last 500 ms stimulus baseline before stimulus onset. We did this calculation separately for each stimulus domain: size, contrast, and orientation. Visually responsive channels were the channels with activity higher than 2.5 
standard deviations of mean baseline activity in at least 4 stimulus conditions (e.g., 4 sizes or 4 orientations).

Frequency analysis was performed using the ft_freqanalysis function from FieldTrip toolbox (Oostenveld et al. 2011). The fast fourier transform (FFT) analysis was performed at every channel and at the single trial level with single taper Hanning window (cfg.method = 'mtmfft' and cfg.taper = 'hann' as an input for ft_freqanalysis). We extracted the power of frequency $1-100 \mathrm{~Hz}$ from the time period $200 \mathrm{~ms}-1200 \mathrm{~ms}$ post-stimulus. Baseline power spectra were calculated with the same method from period $-1000 \mathrm{~ms}$ to $0 \mathrm{~ms}$ pre-stimulus. The power spectrum of each channel was obtained by averaging the single trial power spectra for the tested stimulus condition.

For analysis at the population level, the power spectra from every channel were peak normalized with the following method: first, we identified the strongest power value from all trial-averaged spectra across stimulus conditions. Second, we divided all power spectra to the power from the overall strongest spectrum. This peak normalization procedure also ensured that our results were not disproportionately affected by some channels with particularly strong power values.

For the peak frequency analysis, we identified the frequency with the strongest power for every trial in every condition. The range of frequency considered was $1-100 \mathrm{~Hz}$. Then, we calculated the median of peak frequency. The distribution of peak frequency at all channels was plotted using a MATLAB function plotSpread (Jonas, 2021). The median of the distribution could then be calculated.

To construct tuning curves, we calculated the mean MUA from the same time window we used for frequency analysis, $200 \mathrm{~ms}-1200 \mathrm{~ms}$ post-stimulus. Similar to the frequency analysis, we also performed peak normalization of tuning curves for the population level analysis. For every channel, we identified the stimulus condition with the highest MUA and divided the MUA values at other conditions by the maximum value.

In the orientation tuning experiment, we performed an additional analysis step. Because of different orientation preferences across neural populations, we first determined the orientation preference of each channel. We defined the preferred orientation as the orientation which induced the highest mean MUA at the time window $200 \mathrm{~ms}-1200 \mathrm{~ms}$ after grating onset. This orientation was named orientation $0^{\circ}$ and other orientations were expressed as deviations from $0^{\circ}$. After this centering process, we did the same analysis steps described above.

Microsaccades analysis

We detected microsaccades using an algorithm developed by Engbert and Kliegl ${ }^{49}$. The raw eye traces were first converted to velocities with a moving average window of 5 data samples. Then, on each trial, we computed the threshold of microsaccades detection as 6 times the standard deviation of velocity time series. An event was classified as microsaccades if it exceeded the threshold by at least 3 data samples (minimum duration of $6 \mathrm{~ms})$. We removed all microsaccades with amplitude larger than $1^{\circ}$.

Detection task

The analysis of behavioural data began with removing trials with RT less than $100 \mathrm{~ms}$ or larger than 1000 ms. Next, we binned the trials based on the SOA between stimulus and 
target. The bin was a 50 ms sliding window with an 8.3 ms step. Mean RT was calculated as a function of binned SOA to generate the RT time course. Then we detrended the time course by fitting a second-order polynomial function and removing the fit from the RT time course. We then calculated the FFT of the detrended time course to see if the resulting power spectrum contains a peak in the theta range. To establish statistical significance, we generated a surrogate time course by randomising the RT and SOA from the real, nondetrended time course. This randomisation destroyed the temporal structure, from the original time course. After that, we detrended and calculated FFT from the surrogate time course. This analysis was repeated for 5000 times and for each iteration we obtained the power spectra from the surrogate time course. This results in a surrogate distribution of power value from each frequency. We then set the threshold for statistical significance by taking the 99.67 percentile value from the surrogate distribution for each frequency. The percentile value was corrected for multiple comparisons across 15 frequencies $(1-15 \mathrm{~Hz}), 1$ $(0.05 / 15)=99.67$.

Similar to what we did for the passive viewing task, we analysed the MUA only from visually responsive channels, defined as channels with the first 100 ms MUA higher than at least 2.5 SD of the last $500 \mathrm{~ms}$ of baseline. The frequency analysis for the detection task was performed on 500 - 1500 ms after stimulus onset on successful catch trials only, trials where the target did not occur and the animals maintained fixation for 1500 ms. The reason why we only analyzed the catch trials was to obtain the full stimulus-induced MUA, uninterrupted by the response to target onset and the saccadic response to it.

We tested the relationship between behavioural and neural oscillations by analyzing both measures across sessions $(n=14)$, combined for both monkeys ( $n=10$ for monkey $A L$ and $n=4$ for monkey DP). For each session, we extracted the peak frequency, the frequency with the highest power, separately from RT and MUA power spectrum. All RT peak frequency in each session was statistically significant as defined above. The peak frequency of MUA was obtained from the mean peak normalized power spectra across channels. We then calculated Pearson correlation coefficient between the RT and MUA peak frequency.

\section{Acknowledgement}

This work was supported by starting grant OptoVison 637638 and SNF grant BSET-0_201532 to Michael C Schmid. We thank the Comparative Biology Centre for their help with animal handling and training, Michael Ortiz-Rios for developing the implant method and designing the electrode chambers, and lan Milne with technical assistance for this project. Alex Thiele, Chris Petkov, Alex Maier, Alwin Gieselmann, and Samy Rima for the discussion and advice. Last, we thank J. Anthony Movshon for his constructive comments at the VSS meeting. 


\section{References}

1. VanRullen, R., and Koch, C. (2003). Is perception discrete or continuous? Trends in Cognitive Sciences 7, 207-213.

2. VanRullen, R. (2016). Perceptual Cycles. Trends in Cognitive Sciences.

3. Inhoff, A.W., and Rayner, K. (1986). Parafoveal word processing during eye fixations in reading: Effects of word frequency. Perception \& Psychophysics 40, 431-439.

4. Otero-Millan, J., Troncoso, X.G., Macknik, S.L., Serrano-Pedraza, I., and Martinez-Conde, S. (2008). Saccades and microsaccades during visual fixation, exploration, and search: Foundations for a common saccadic generator. Journal of Vision 8, 1-18.

5. Bosman, C.A., Womelsdorf, T., Desimone, R., and Fries, P. (2009). A microsaccadic rhythm modulates gamma-band synchronization and behavior. Journal of Neuroscience 29, 9471-9480.

6. Lowet, E., Roberts, M.J., Bosman, C.A., Fries, P., and de Weerd, P. (2016). Areas V1 and V2 show microsaccade-related $3-4-\mathrm{Hz}$ covariation in gamma power and frequency. European Journal of Neuroscience 43, 1286-1296.

7. Hafed, Z.M., and Ignashchenkova, A. (2013). On the Dissociation between Microsaccade Rate and Direction after Peripheral Cues: Microsaccadic Inhibition Revisited. J. Neurosci. 33, 16220-16235.

8. Landau, A.N., and Fries, P. (2012). Attention samples stimuli rhythmically. Current Biology.

9. Fiebelkorn, I.C., Saalmann, Y.B., and Kastner, S. (2013). Rhythmic sampling within and between objects despite sustained attention at a cued location. Current Biology.

10. Michel, R., Dugué, L., and Busch, N.A. (2021). Distinct contributions of alpha and theta rhythms to perceptual and attentional sampling. European Journal of Neuroscience.

11. Song, K., Meng, M., Chen, L., Zhou, K., and Luo, H. (2014). Behavioral Oscillations in Attention: Rhythmic Pulses Mediated through Band. Journal of Neuroscience.

12. Dugué, L., Roberts, M., and Carrasco, M. (2016). Attention Reorients Periodically. Current Biology 26, 1595-1601.

13. Re, D., Inbar, M., Richter, C.G., and Landau, A.N. (2019). Feature-Based Attention Samples Stimuli Rhythmically. Current Biology 29, 693-699.e4.

14. Hafed, Z.M., Chen, C.-Y., and Tian, X. (2015). Vision, Perception, and Attention through the Lens of Microsaccades: Mechanisms and Implications. Frontiers in Systems Neuroscience 9, 167.

15. VanRullen, R. (2016). Perceptual Cycles. Trends in Cognitive Sciences. 
16. Hafed, Z.M. (2013). Alteration of Visual Perception prior to Microsaccades. Neuron 77, 775-786.

17. Busch, N.A., Dubois, J., and VanRullen, R. (2009). The Phase of Ongoing EEG Oscillations Predicts Visual Perception. Journal of Neuroscience.

18. Busch, N.A., and VanRullen, R. (2010). Spontaneous EEG oscillations reveal periodic sampling of visual attention. Proceedings of the National Academy of Sciences.

19. Drewes, J., and VanRullen, R. (2011). This Is the Rhythm of Your Eyes: The Phase of Ongoing Electroencephalogram Oscillations Modulates Saccadic Reaction Time. Journal of Neuroscience.

20. Dugué, L., Marque, P., and VanRullen, R. (2015). Theta Oscillations Modulate Attentional Search Performance Periodically. Journal of Cognitive Neuroscience 27, 945-958.

21. Hanslmayr, S., Volberg, G., Wimber, M., Dalal, S.S., and Greenlee, M.W. (2013). Prestimulus Oscillatory Phase at $7 \mathrm{~Hz}$ Gates Cortical Information Flow and Visual Perception. Current Biology 23, 2273-2278.

22. Gaillard, C., Ben Hadj Hassen, S., Di Bello, F., Bihan-Poudec, Y., VanRullen, R., and Ben Hamed, S. (2020). Prefrontal attentional saccades explore space rhythmically. Nature Communications.

23. Fiebelkorn, I.C., and Kastner, S. (2021). Spike Timing in the Attention Network Predicts Behavioral Outcome Prior to Target Selection. Neuron 109, 177-188.e4.

24. Fiebelkorn, I.C., Pinsk, M.A., and Kastner, S. (2018). A Dynamic Interplay within the Frontoparietal Network Underlies Rhythmic Spatial Attention. Neuron.

25. Fiebelkorn, I.C., Pinsk, M.A., and Kastner, S. (2019). The mediodorsal pulvinar coordinates the macaque fronto-parietal network during rhythmic spatial attention. Nature Communications.

26. Helfrich, R.F., Fiebelkorn, I.C., Szczepanski, S.M., Lin, J.J., Parvizi, J., Knight, R.T., and Kastner, S. (2018). Neural Mechanisms of Sustained Attention Are Rhythmic. Neuron.

27. Noudoost, B., Chang, M.H., Steinmetz, N.A., and Moore, T. (2010). Top-down control of visual attention. Current Opinion in Neurobiology 20, 183-190.

28. Fiebelkorn, I.C., and Kastner, S. (2019). A Rhythmic Theory of Attention. Trends in Cognitive Sciences 23, 87-101.

29. Nakamura, K., Mikami, A., and Kubota, K. (1991). Unique oscillatory activity related to visual processing in the temporal pole of monkeys. Neuroscience Research 12, 293-299.

30. Nakamura, K., Mikami, A., and Kubota, K. (1992). Oscillatory neuronal activity related to visual short-term memory in monkey temporal pole. NeuroReport. 
31. Sheinberg, D.L., and Logothetis, N.K. (1997). The role of temporal cortical areas in perceptual organization. Neurobiology.

32. Rollenhagen, J.E., and Olson, C.R. (2005). Low-frequency oscillations arising from competitive interactions between visual stimuli in macaque inferotemporal cortex. Journal of neurophysiology.

33. Lee, H., Simpson, G. V., Logothetis, N.K., and Rainer, G. (2005). Phase locking of single neuron activity to theta oscillations during working memory in monkey extrastriate visual cortex. Neuron.

34. Kienitz, R., Schmiedt, J.T., Shapcott, K.A., Kouroupaki, K., Saunders, R.C., and Schmid, M.C. (2018). Theta Rhythmic Neuronal Activity and Reaction Times Arising from Cortical Receptive Field Interactions during Distributed Attention. Current Biology.

35. Cox, M.A., Schmid, M.C., Peters, A.J., Saunders, R.C., Leopold, D.A., and Maier, A. (2013). Receptive field focus of visual area $\mathrm{V} 4$ neurons determines responses to illusory surfaces. Proceedings of the National Academy of Sciences.

36. Bastos, A.M., Vezoli, J., Bosman, C.A., Schoffelen, J.M., Oostenveld, R., Dowdall, J.R., DeWeerd, P., Kennedy, H., and Fries, P. (2015). Visual areas exert feedforward and feedback influences through distinct frequency channels. Neuron.

37. Spyropoulos, G., Bosman, C.A., and Fries, P. (2018). A theta rhythm in macaque visual cortex and its attentional modulation. Proceedings of the National Academy of Sciences, 201719433.

38. Kienitz, R., Cox, M.A., Dougherty, K., Saunders, R.C., Schmiedt, J.T., Leopold, D.A., Maier, A., and Schmid, M.C. (2020). Theta, but Not Gamma Oscillations in Area V4 Depend on Input from Primary Visual Cortex. Current Biology.

39. Carandini, M., Demb, J.B., Mante, V., Tolhurst, D.J., Dan, Y., Olshausen, B.A., Gallant, J.L., and Rust, N.C. (2005). Do we know what the early visual system does? In Journal of Neuroscience (Society for Neuroscience), pp. 10577-10597.

40. Foster, K.H., Gaska, J.P., Nagler, M., and Pollen, D.A. (1985). Spatial and temporal frequency selectivity of neurones in visual cortical areas V1 and V2 of the macaque monkey. The Journal of Physiology 365, 331-363.

41. Hawken, M.J., Shapley, R.M., and Grosof, D.H. (1996). Temporal-frequency selectivity in monkey visual cortex. Visual Neuroscience 13, 477-492.

42. Jones, H.E., Grieve, K.L., Wang, W., and Sillito, A.M. (2001). Surround Suppression in Primate V1. Journal of Neurophysiology 86, 2011-2028.

43. Cavanaugh, J.R., Bair, W., and Movshon, J.A. (2002). Nature and Interaction of Signals From the Receptive Field Center and Surround in Macaque V1 Neurons. Journal of Neurophysiology 88, 2530-2546. 
44. Albrecht, D.G., and Hamilton, D.B. (1982). Striate cortex of monkey and cat: contrast response function. Journal of Neurophysiology 48, 217-237.

45. Priebe, N.J. (2016). Mechanisms of Orientation Selectivity in the Primary Visual Cortex. Annual Review of Vision Science 2, 85-107.

46. Leopold, D.A., and Logothetis, N.K. (1998). Microsaccades differentially modulate neural activity in the striate and extrastriate visual cortex. Experimental Brain Research 123, 341-345.

47. Martinez-Conde, S., Macknik, S.L., and Hubel, D.H. (2000). Microsaccadic eye movements and firing of single cells in the striate cortex of macaque monkeys. Nature Neuroscience 3, 251-258.

48. Troncoso, X.G., McCamy, M.B., Jazi, A.N., Cui, J., Otero-Millan, J., MacKnik, S.L., Costela, F.M., and Martinez-Conde, S. (2015). V1 neurons respond differently to object motion versus motion from eye movements. Nature Communications 6, 1-10.

49. Engbert, R., and Kliegl, R. (2003). Microsaccades uncover the orientation of covert attention. Vision Research 43, 1035-1045.

50. Sheinberg, D.L., and Logothetis, N.K. (1997). The role of temporal cortical areas in perceptual organization. Neurobiology.

51. Cox, M.A., Schmid, M.C., Peters, A.J., Saunders, R.C., Leopold, D.A., and Maier, A. (2013). Receptive field focus of visual area $\mathrm{V} 4$ neurons determines responses to illusory surfaces. Proceedings of the National Academy of Sciences.

52. Allison, J.D., Smith, K.R., and Bonds, A.B. (2001). Temporal-frequency tuning of crossorientation suppression in the cat striate cortex. Visual Neuroscience 18, 941-948.

53. Yu, H.-H., Verma, R., Yang, Y., Tibballs, H.A., Lui, L.L., Reser, D.H., and Rosa, M.G.P. (2010). Spatial and temporal frequency tuning in striate cortex: functional uniformity and specializations related to receptive field eccentricity. European Journal of Neuroscience 31, 1043-1062.

54. Singh, K.D., Smith, A.T., and Greenlee, M.W. (2000). Spatiotemporal Frequency and Direction Sensitivities of Human Visual Areas Measured Using fMRI. Neurolmage 12, 550-564.

55. Singh, M., Kim, S., and Kim, T.-S. (2003). Correlation between BOLD-fMRI and EEG signal changes in response to visual stimulus frequency in humans. Magnetic Resonance in Medicine 49, 108-114.

56. Himmelberg, M.M., and Wade, A.R. (2019). Eccentricity-dependent temporal contrast tuning in human visual cortex measured with fMRI. Neurolmage 184, 462-474.

57. Chai, Y., Handwerker, D.A., Marrett, S., Gonzalez-Castillo, J., Merriam, E.P., Hall, A., Molfese, P.J., and Bandettini, P.A. (2019). Visual temporal frequency preference shows a distinct cortical architecture using fMRI. Neurolmage 197, 13-23. 
58. Fawcett, I.P., Barnes, G.R., Hillebrand, A., and Singh, K.D. (2004). The temporal frequency tuning of human visual cortex investigated using synthetic aperture magnetometry. Neurolmage 21, 1542-1553.

59. Fylan, F., Holliday, I.E., Singh, K.D., Anderson, S.J., and Harding, G.F.A. (1997). Magnetoencephalographic Investigation of Human Cortical Area V1 Using Color Stimuli. Neurolmage 6, 47-57.

60. Gieselmann, M.A., and Thiele, A. (2008). Comparison of spatial integration and surround suppression characteristics in spiking activity and the local field potential in macaque V1. European Journal of Neuroscience 28, 447-459.

61. Lima, B., Singer, W., Chen, N.H., and Neuenschwander, S. (2010). Synchronization dynamics in response to plaid stimuli in monkey V1. Cerebral Cortex 20, 1556-1573.

62. Ray, S., and Maunsell, J.H.R. (2011). Different Origins of Gamma Rhythm and HighGamma Activity in Macaque Visual Cortex. PLOS Biology 9, e1000610.

63. Jia, X., Xing, D., and Kohn, A. (2013). No Consistent Relationship between Gamma Power and Peak Frequency in Macaque Primary Visual Cortex. J. Neurosci. 33, 17-25.

64. van Pelt, S., and Fries, P. (2013). Visual stimulus eccentricity affects human gamma peak frequency. Neurolmage 78, 439-447.

65. Martinez-Conde, S., Otero-Millan, J., and Macknik, S.L. (2013). The impact of microsaccades on vision: towards a unified theory of saccadic function. Nat Rev Neurosci 14, 83-96.

66. Poletti, M., and Rucci, M. (2016). A compact field guide to the study of microsaccades: Challenges and functions. Vision Research 118, 83-97.

67. Gao, X., Yan, H., and Sun, H. (2015). Modulation of microsaccade rate by task difficulty revealed through between- and within-trial comparisons. Journal of Vision 15, 3.

68. Fiebelkorn, I.C., Saalmann, Y.B., and Kastner, S. (2013). Rhythmic sampling within and between objects despite sustained attention at a cued location. Current Biology.

69. Michel, R., Dugué, L., and Busch, N.A. (2021). Distinct contributions of alpha and theta rhythms to perceptual and attentional sampling. European Journal of Neuroscience.

70. Helfrich, R.F., Fiebelkorn, I.C., Szczepanski, S.M., Lin, J.J., Parvizi, J., Knight, R.T., and Kastner, S. (2018). Neural Mechanisms of Sustained Attention Are Rhythmic. Neuron.

71. Gaillard, C., Ben Hadj Hassen, S., Di Bello, F., Bihan-Poudec, Y., VanRullen, R., and Ben Hamed, S. (2020). Prefrontal attentional saccades explore space rhythmically. Nature Communications.

72. Dugué, L., Beck, A.-A., Marque, P., and VanRullen, R. (2019). Contribution of FEF to Attentional Periodicity during Visual Search: A TMS Study. eNeuro 6. 
73. Squire, R.F., Noudoost, B., Schafer, R.J., and Moore, T. (2013). Prefrontal Contributions to Visual Selective Attention. Annual Review of Neuroscience 36, 451-466.

74. Bisley, J.W., and Goldberg, M.E. (2010). Attention, Intention, and Priority in the Parietal Lobe. Annual Review of Neuroscience 33, 1-21.

75. Dugué, L., Roberts, M., and Carrasco, M. (2016). Attention Reorients Periodically. Current Biology 26, 1595-1601.

76. Posner, M.I. (1980). Orienting of Attention. Quarterly Journal of Experimental Psychology 32, 3-25.

77. VanRullen, R. (2018). Attention Cycles. Neuron.

78. Gray, H., Bertrand, H., Mindus, C., Flecknell, P., Rowe, C., and Thiele, A. (2016). Physiological, Behavioral, and Scientific Impact of Different Fluid Control Protocols in the Rhesus Macaque (Macaca mulatta). eNeuro 3.

79. Oostenveld, R., Fries, P., Maris, E., and Schoffelen, J.-M. (2011). FieldTrip: Open Source Software for Advanced Analysis of MEG, EEG, and Invasive Electrophysiological Data. Computational Intelligence and Neuroscience 2011, 1-9.

80. Supèr, H., and Roelfsema, P.R. (2005). Chronic multiunit recordings in behaving animals: Advantages and limitations. Progress in Brain Research 147, 263-282. 
A

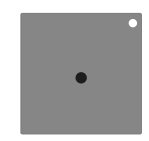
$0.3^{\circ}$
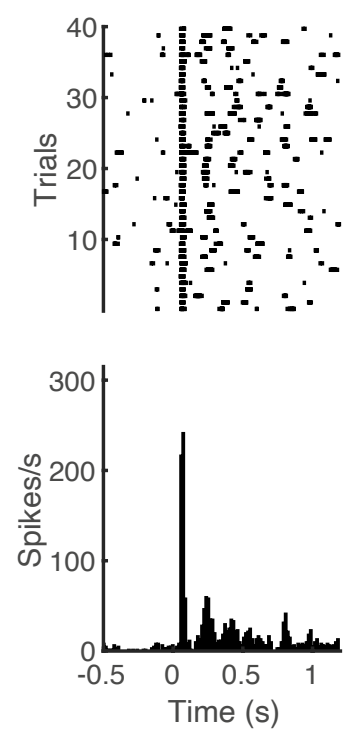

B

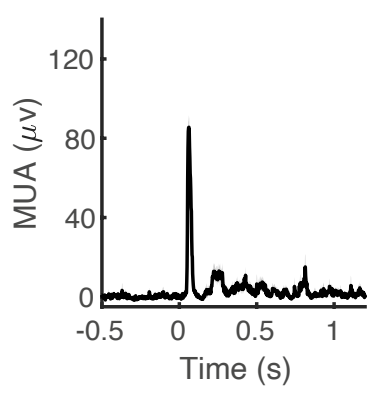

C

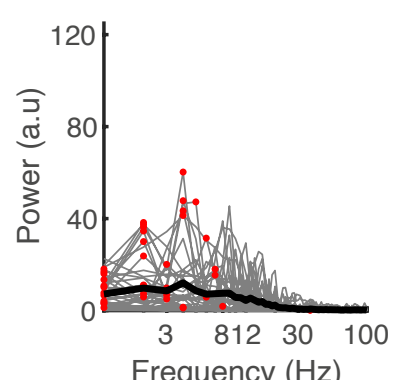

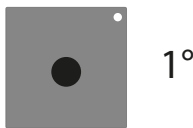
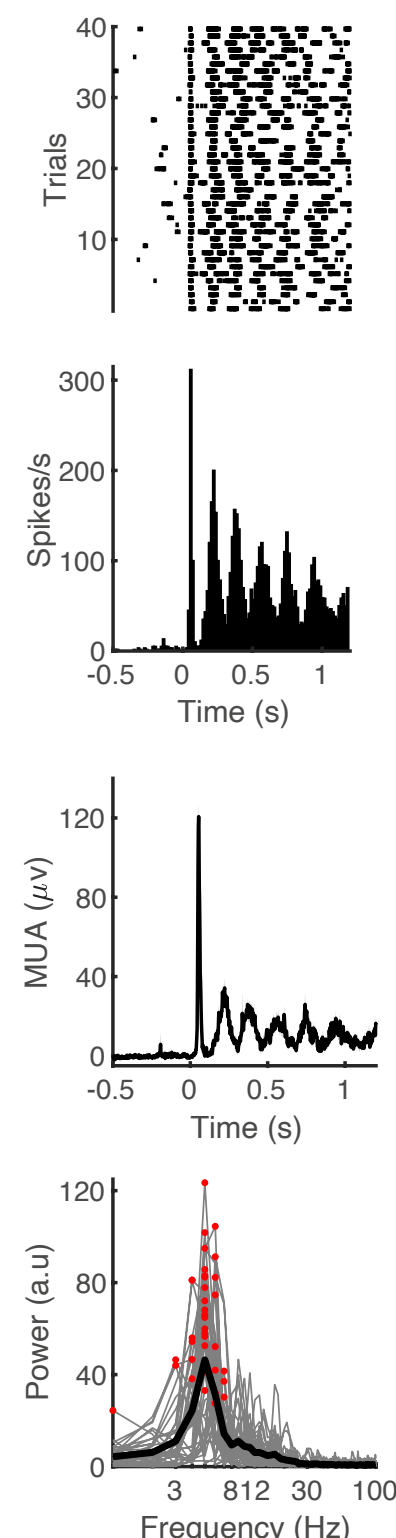

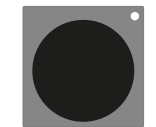

$4^{\circ}$
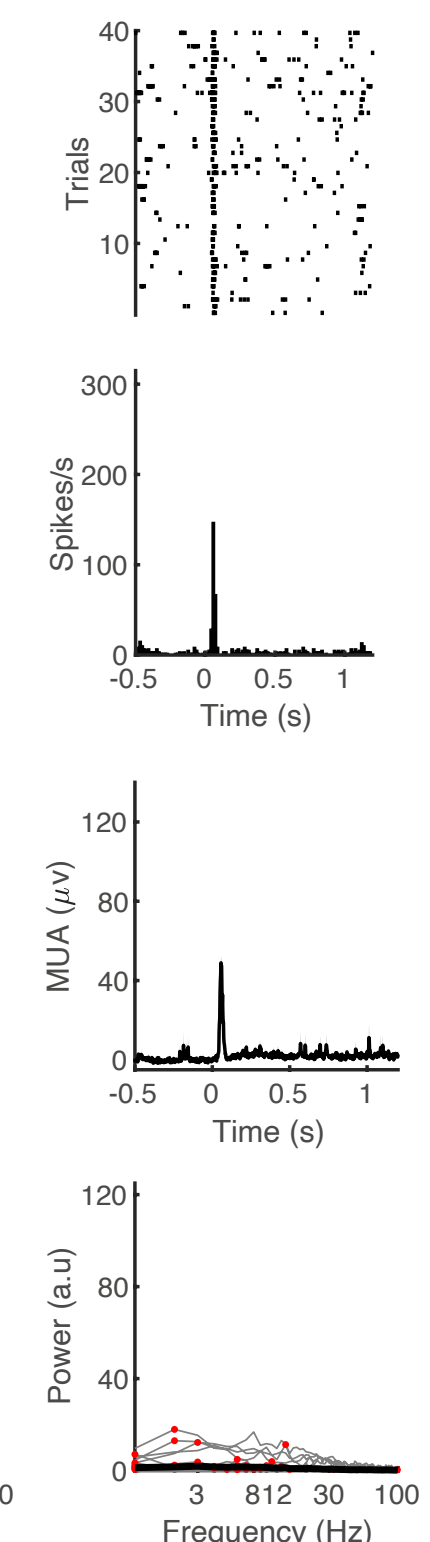

D

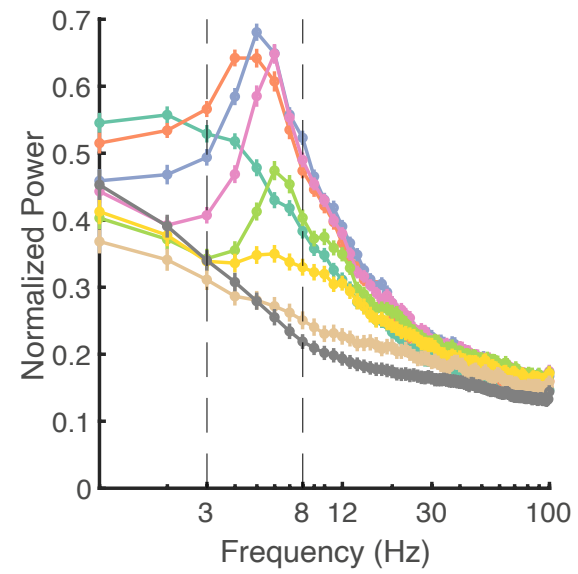

E

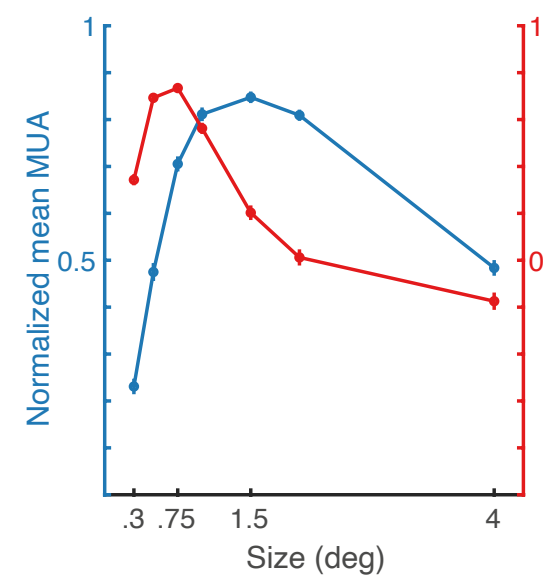

F

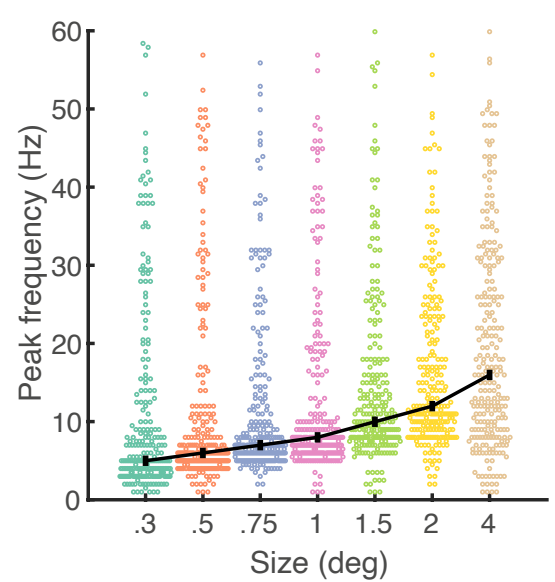

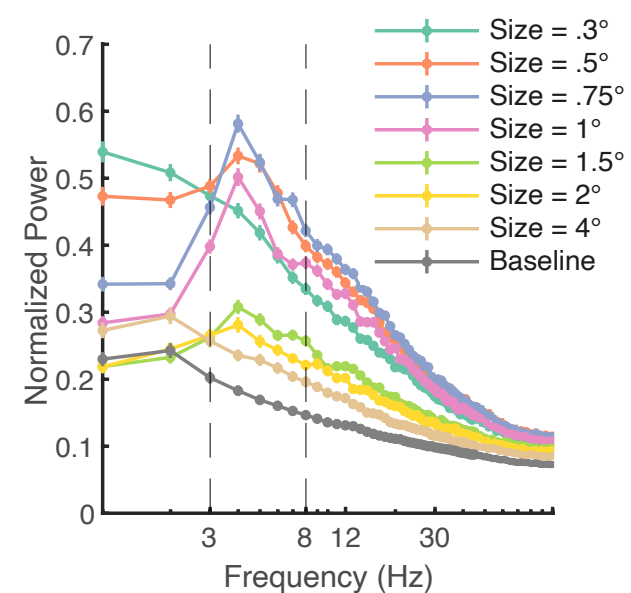
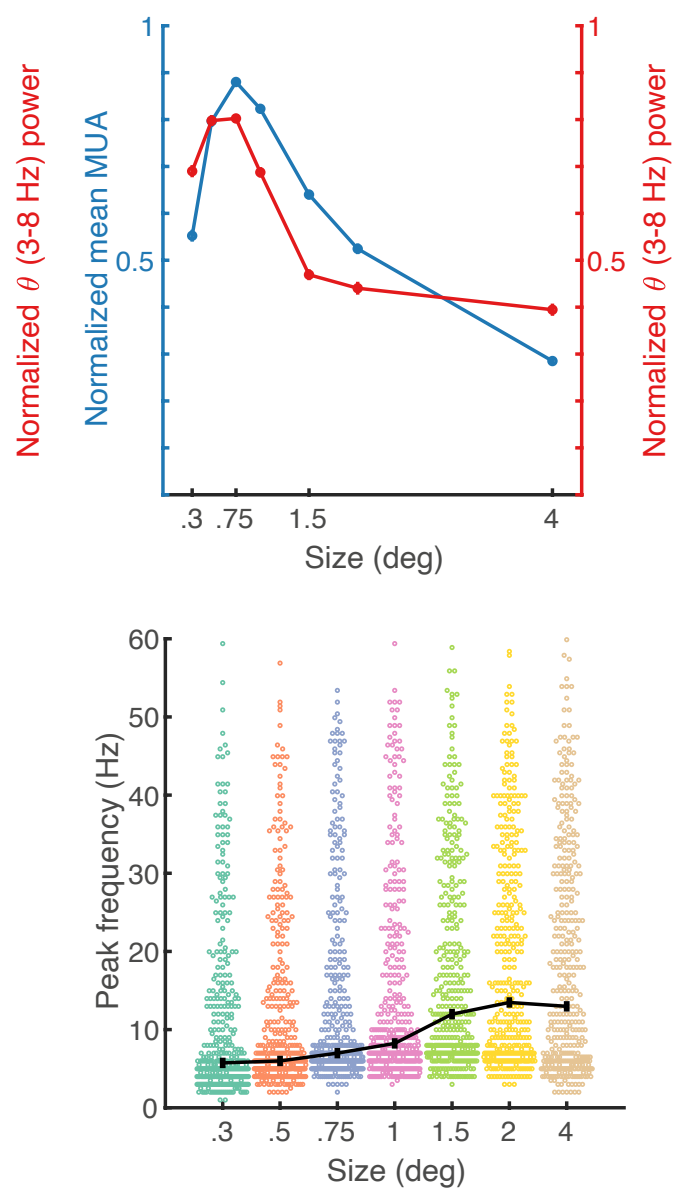


\section{FIGURE 2}

A

Stimulus $\mathrm{X}$ coordinate $\left({ }^{\circ}\right)$

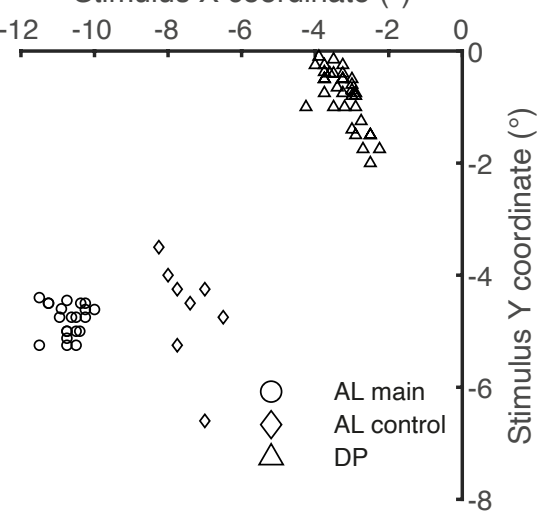

B

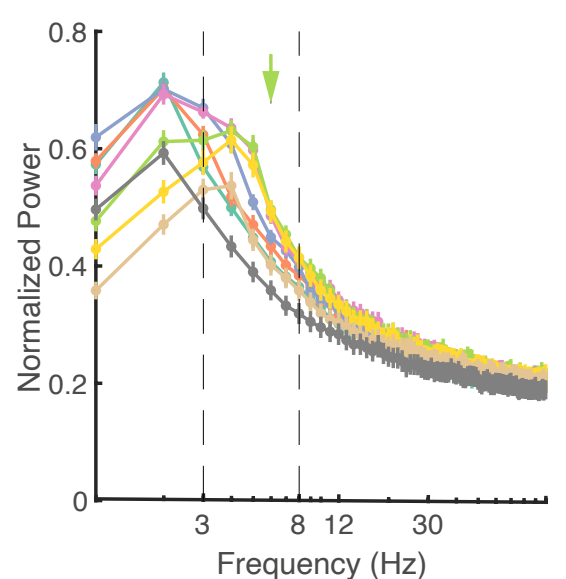

C

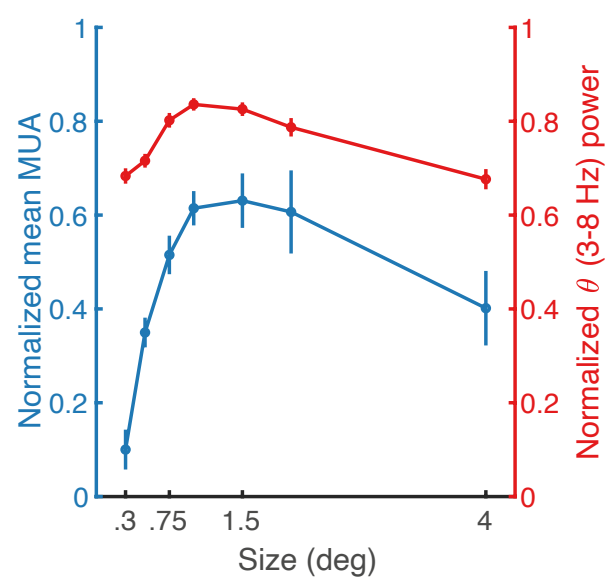

D

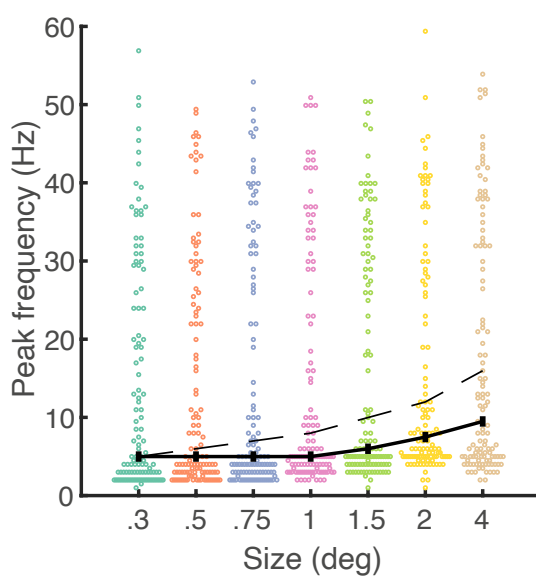


FIGURE 3

A

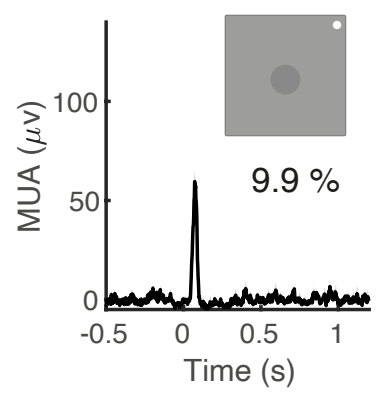

B

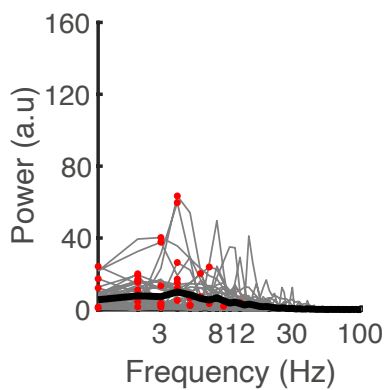

C
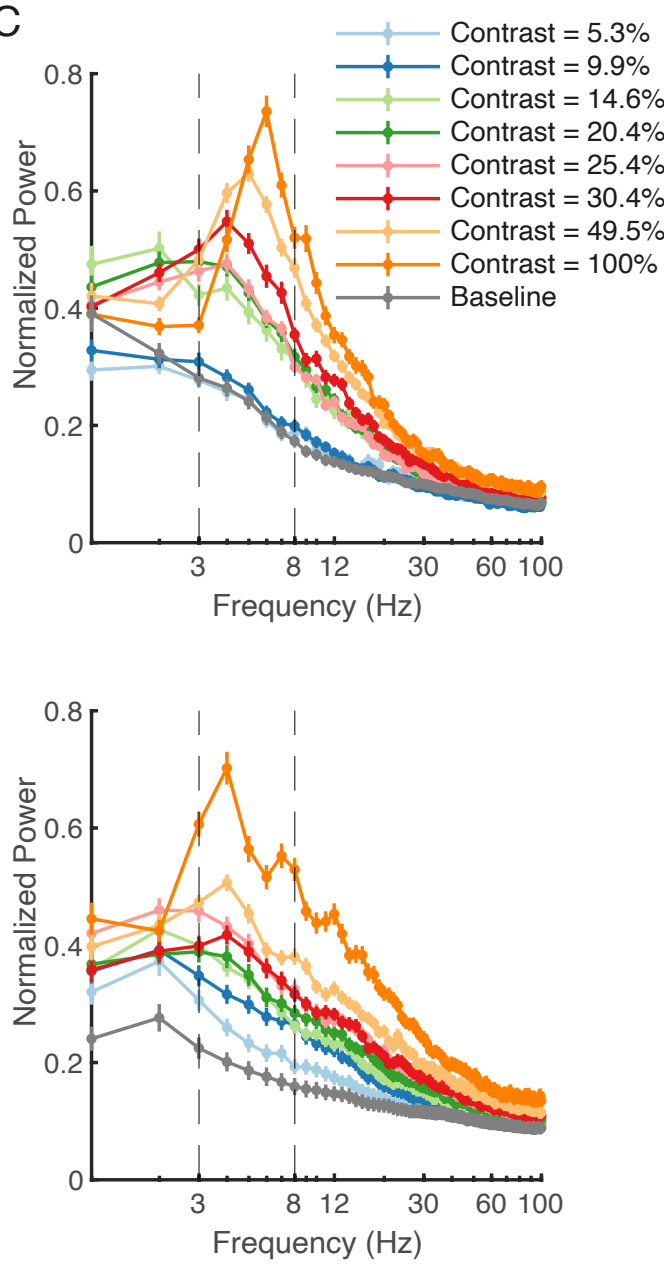
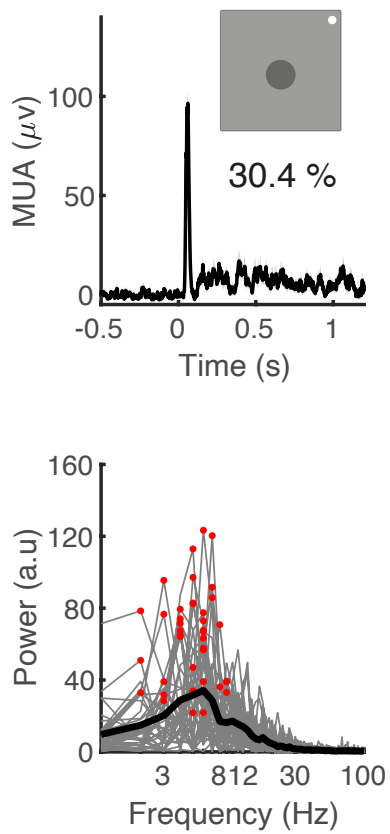

D
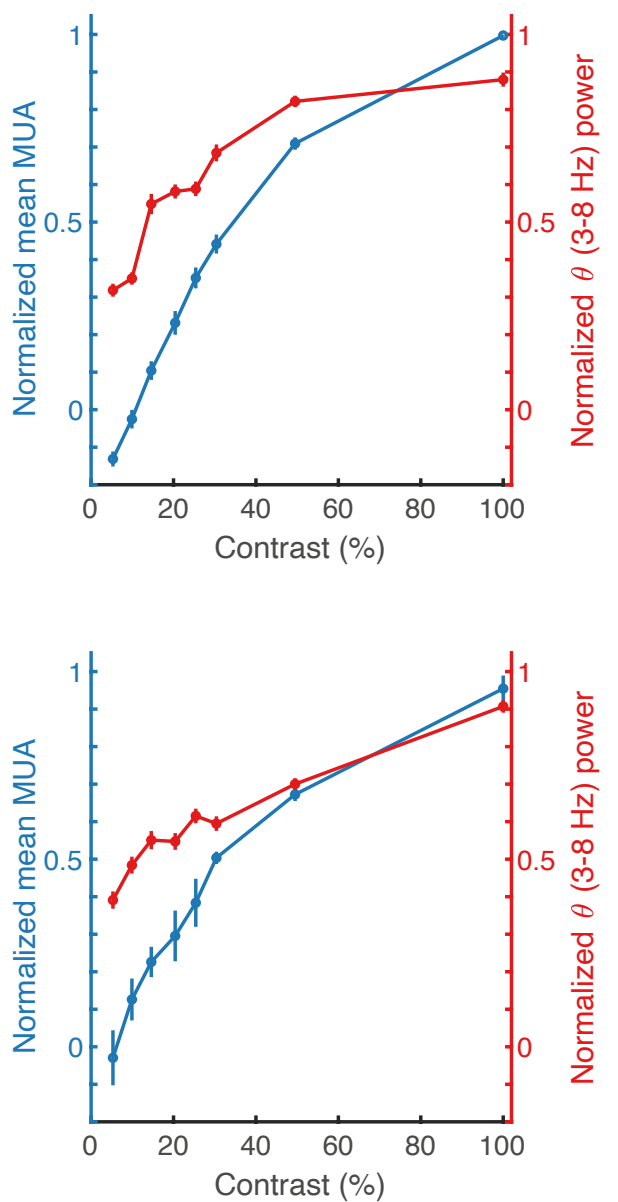
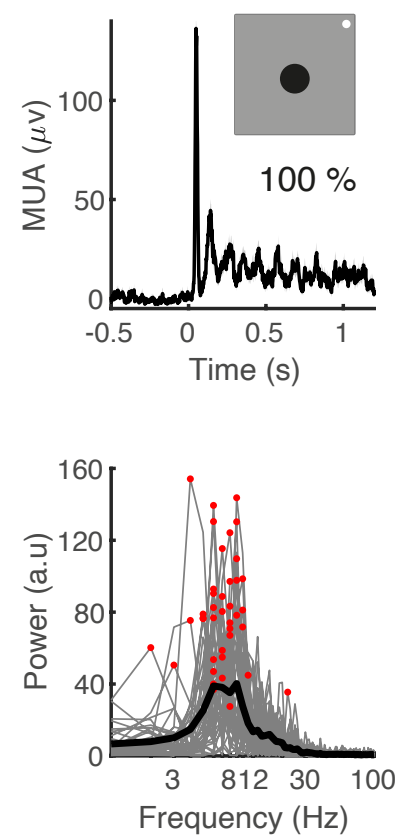

E
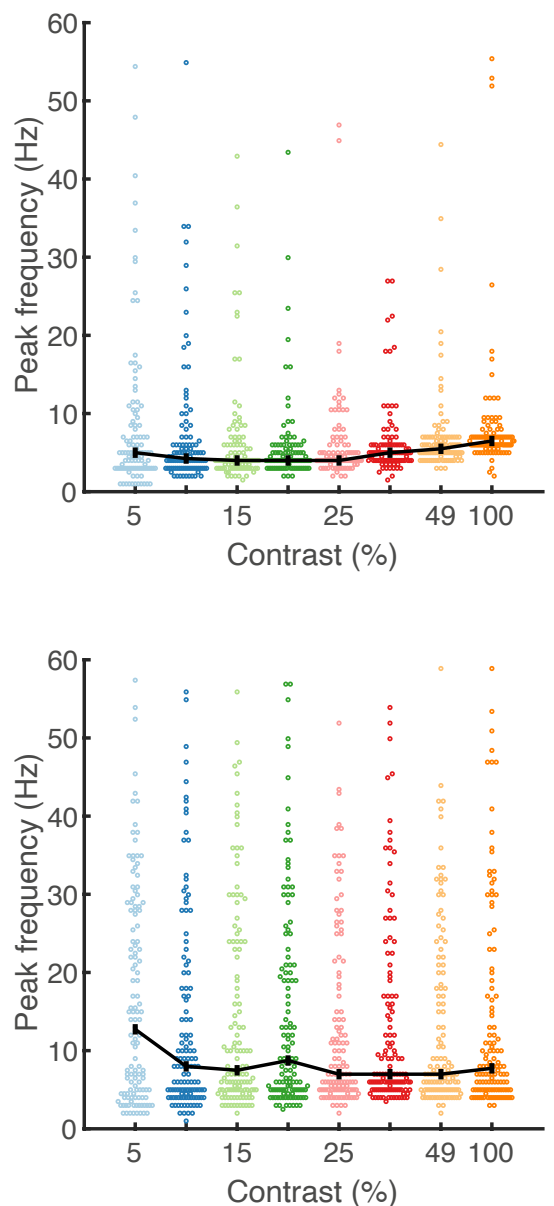
A

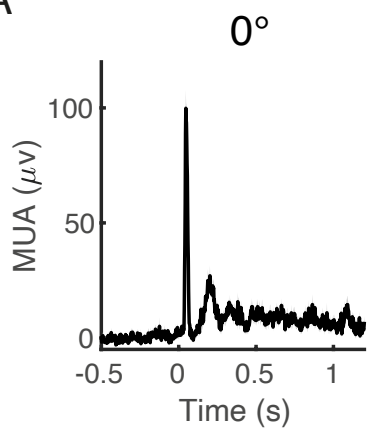

B
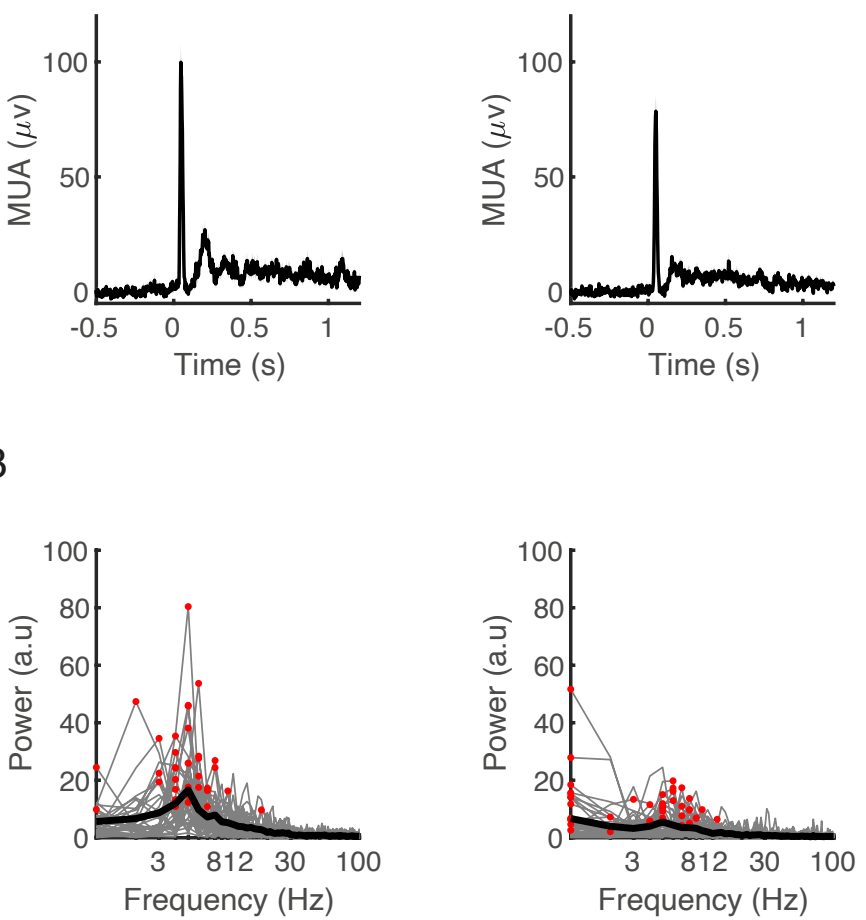

$162^{\circ}$
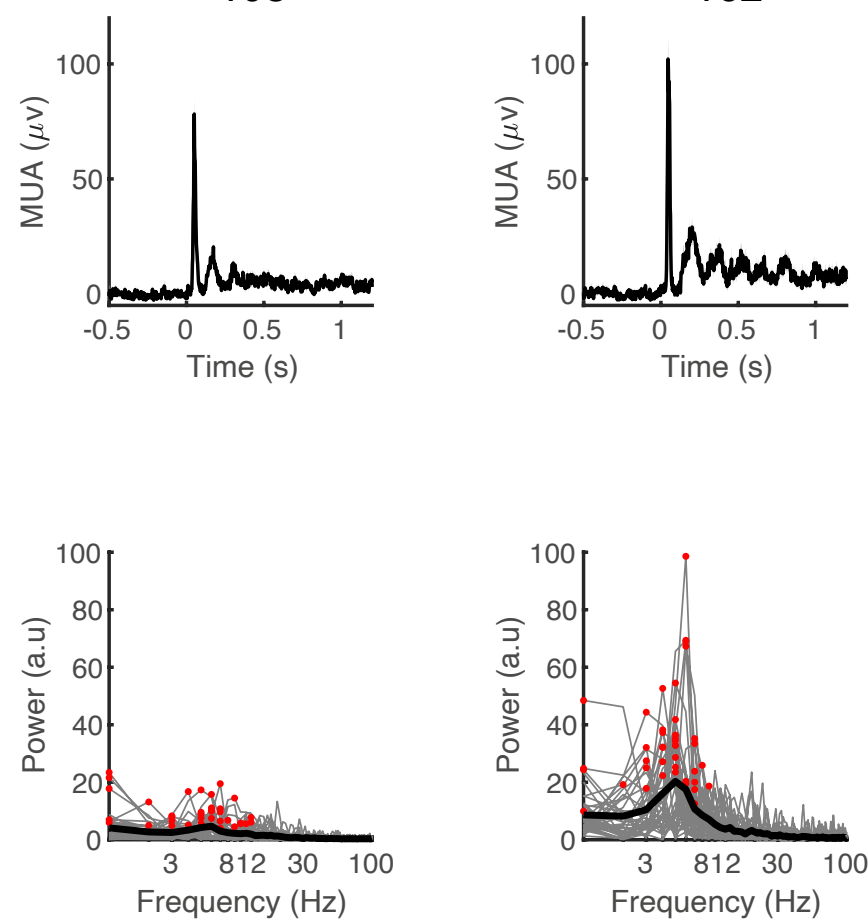

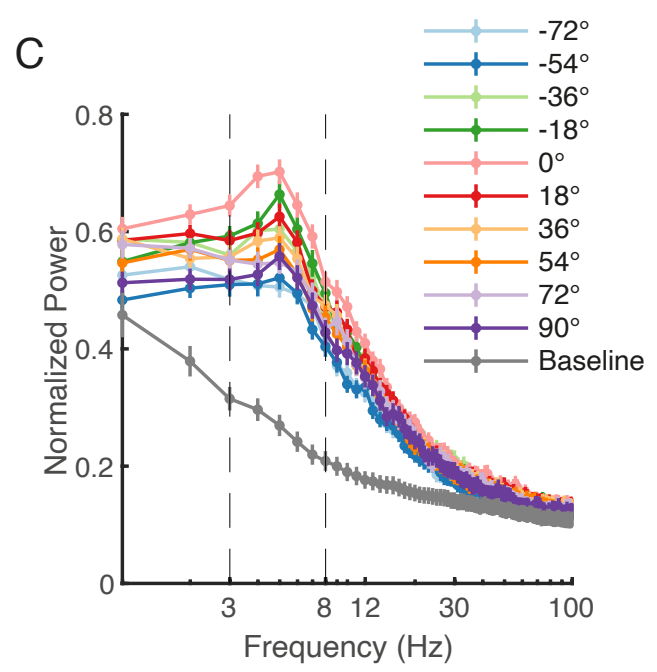

D
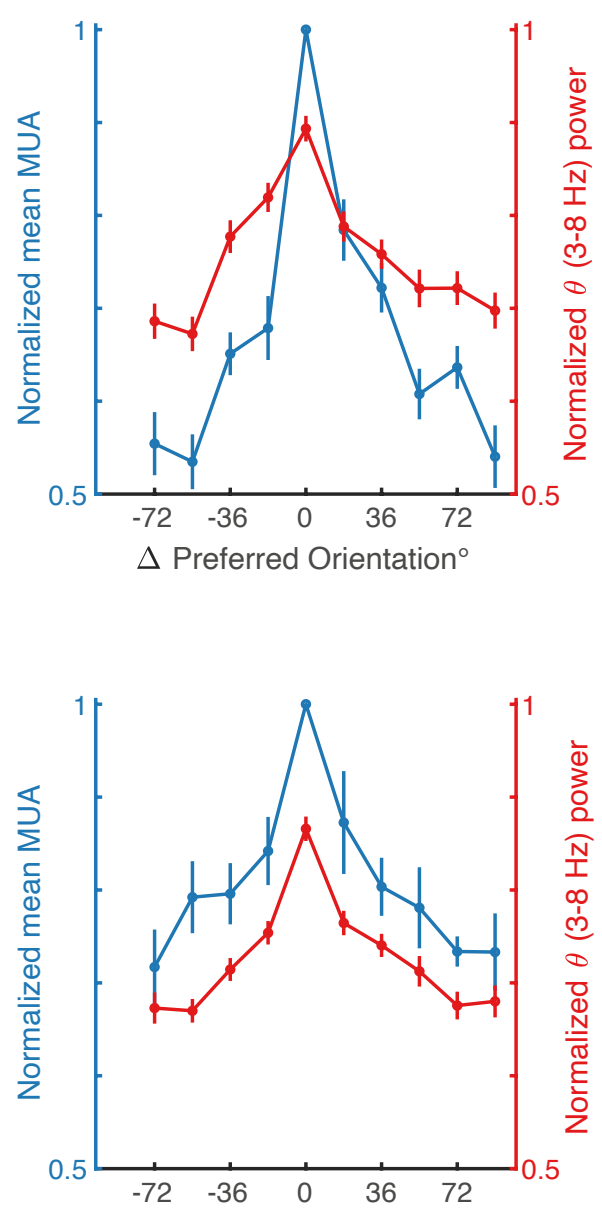

$\Delta$ Preferred Orientation ${ }^{\circ}$
E
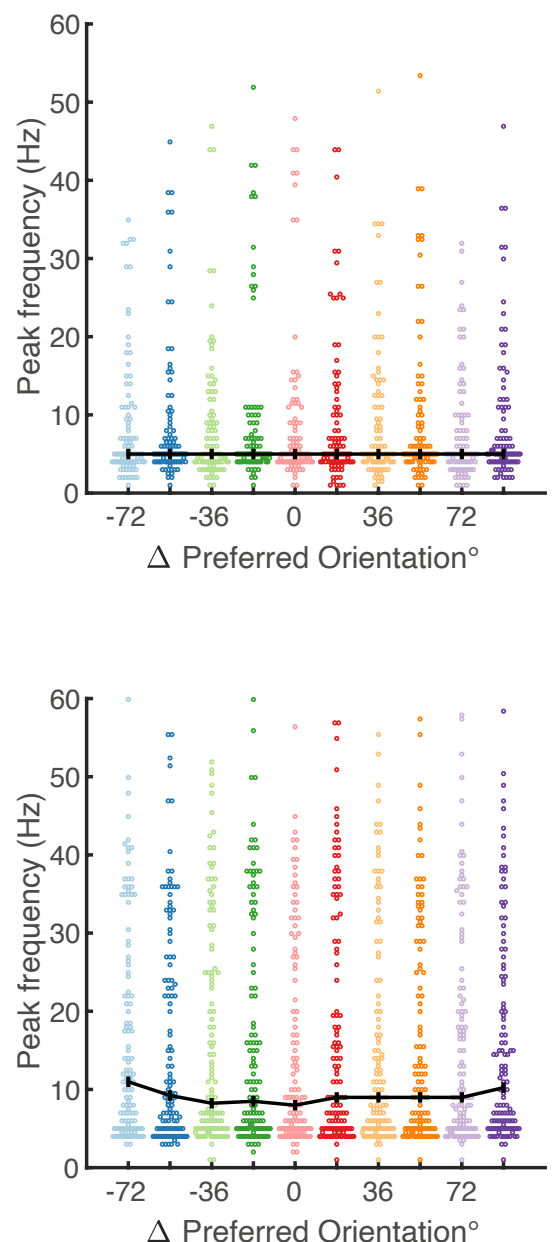


\section{FIGURE 5}

Monkey AL

A

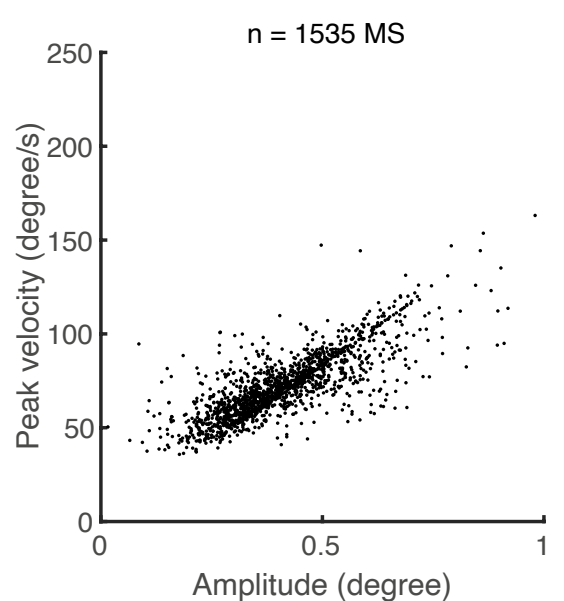

C

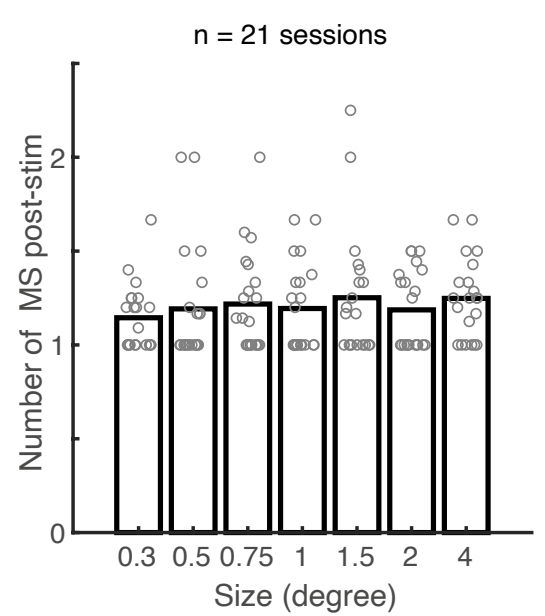

E

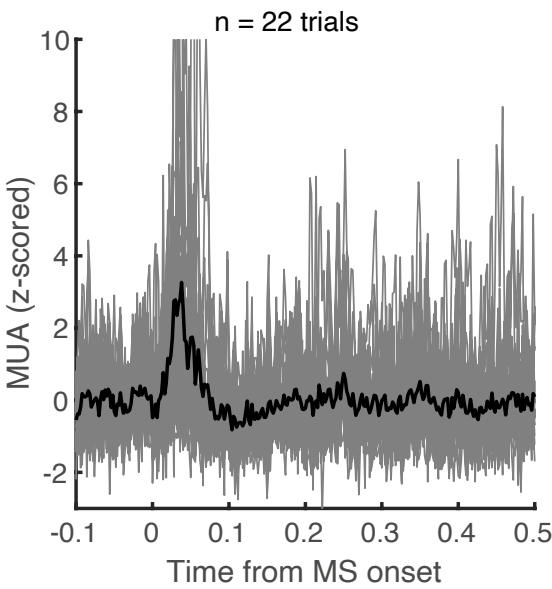

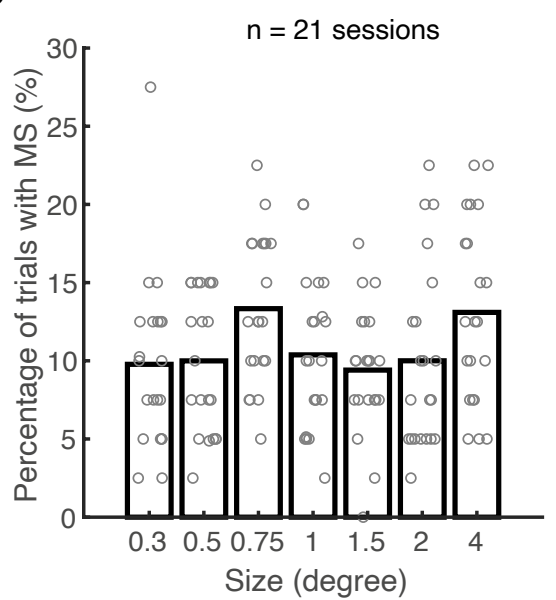

D

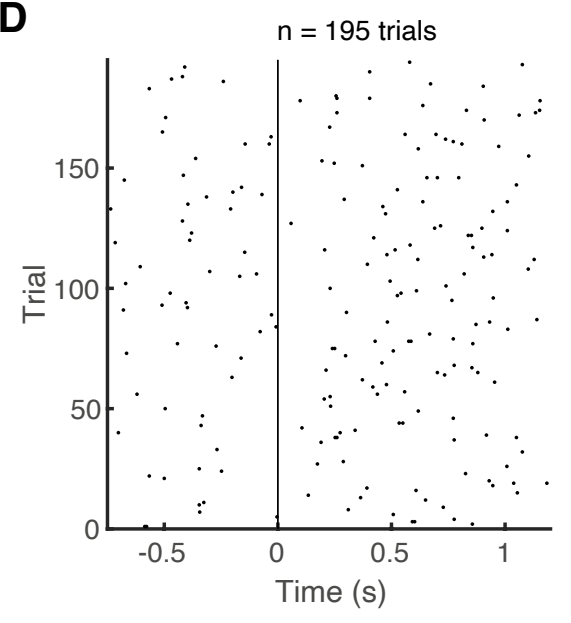

F

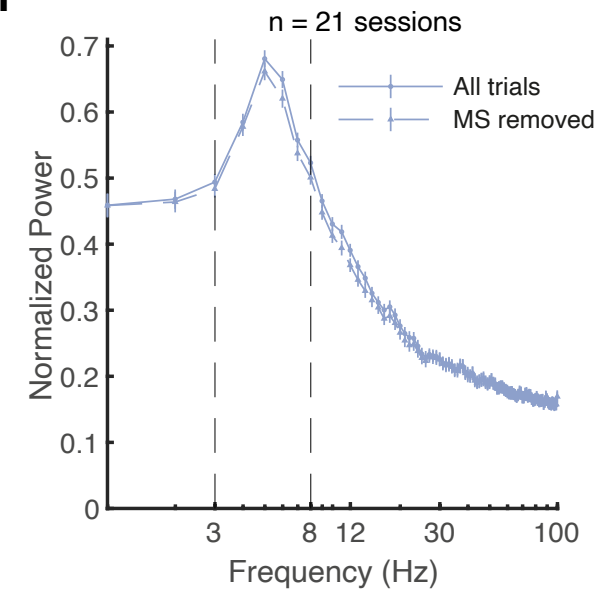

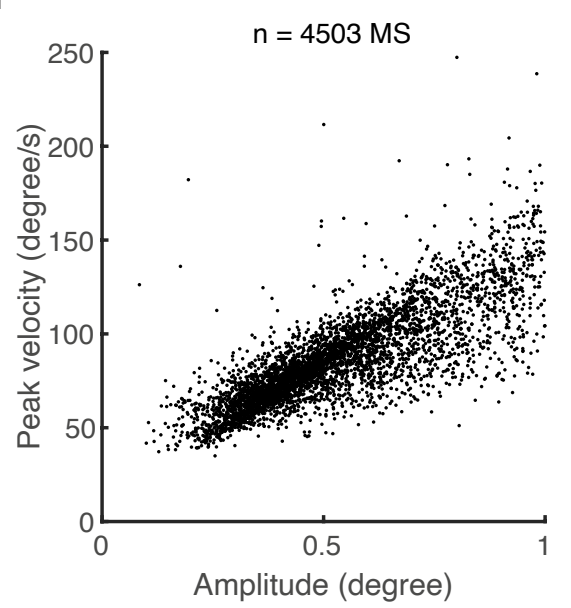

I

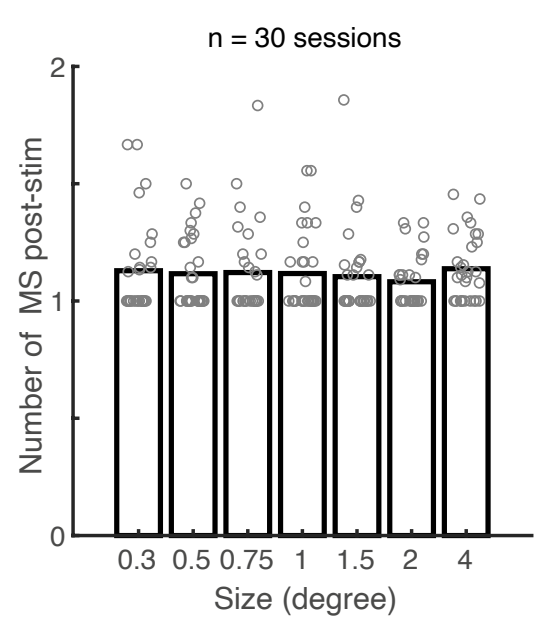

K

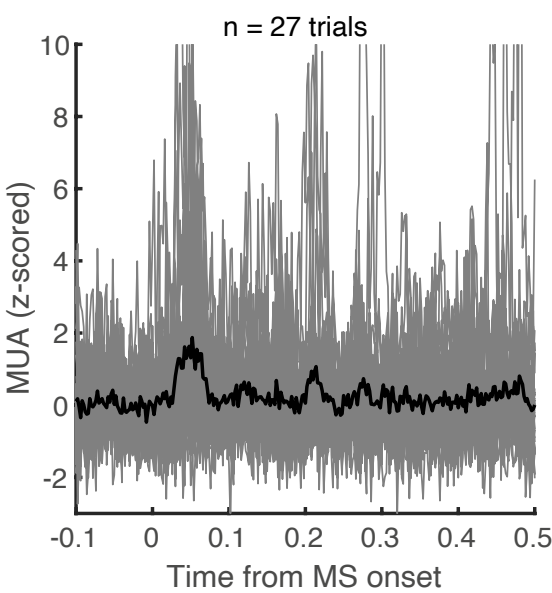

Monkey DP

H

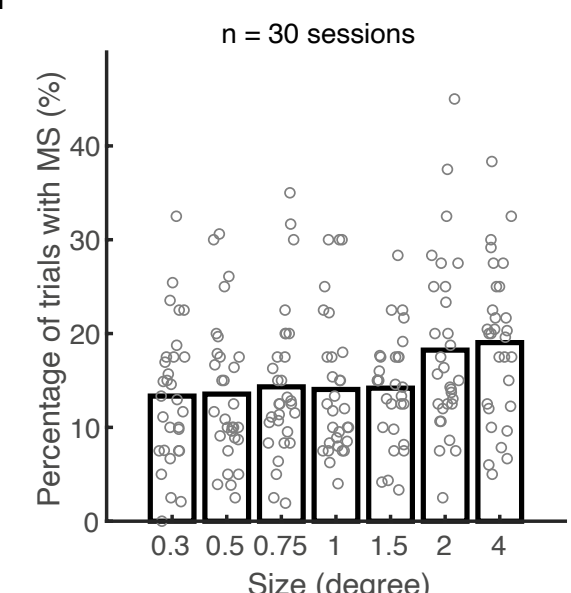

J

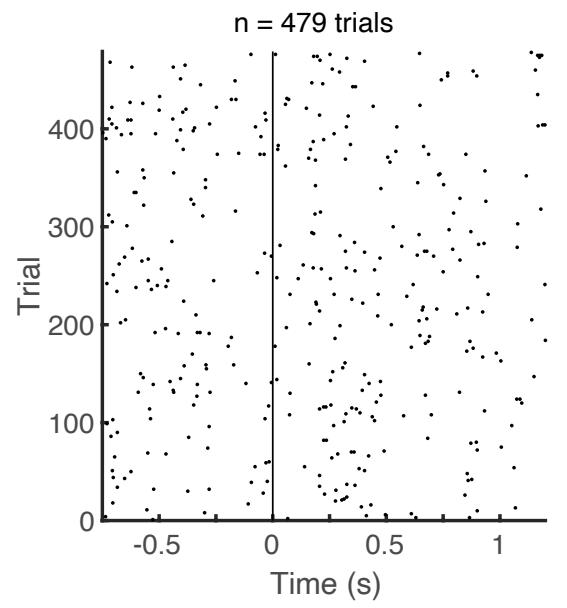

L

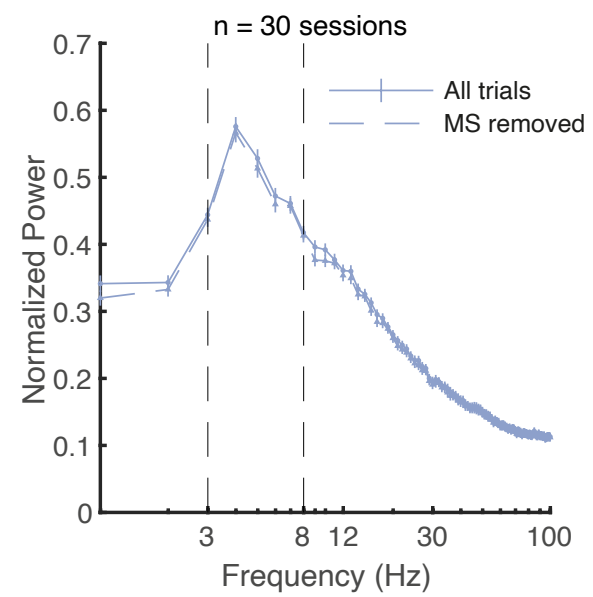




\section{FIGURE 6}

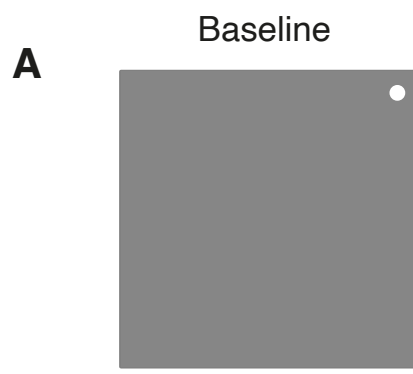

Stimulus on

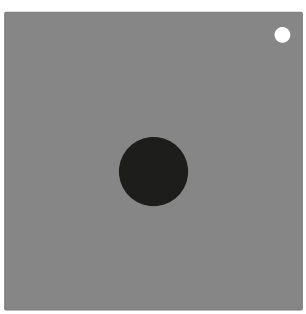

$1000 \mathrm{~ms}$

\section{$500-1500 \mathrm{~ms}$}

Target on

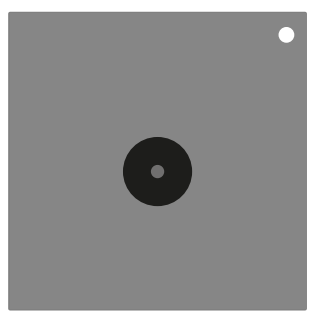

$1000 \mathrm{~ms}$
Monkey AL

B

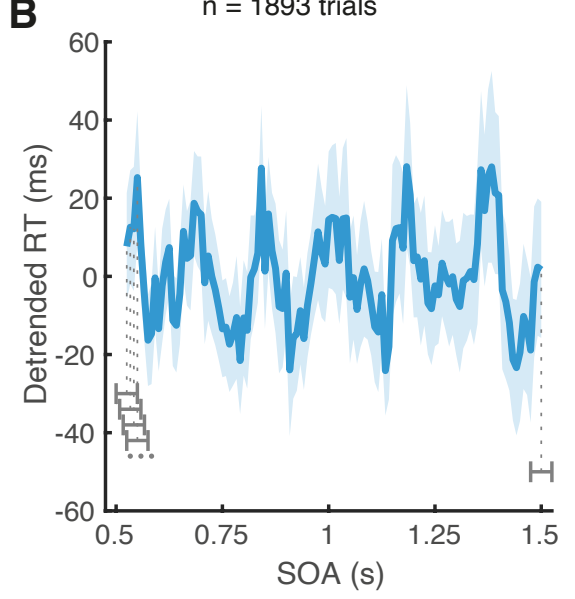

C $\mathrm{n}_{\mathrm{MUA}}=565$ trials; 14 channels

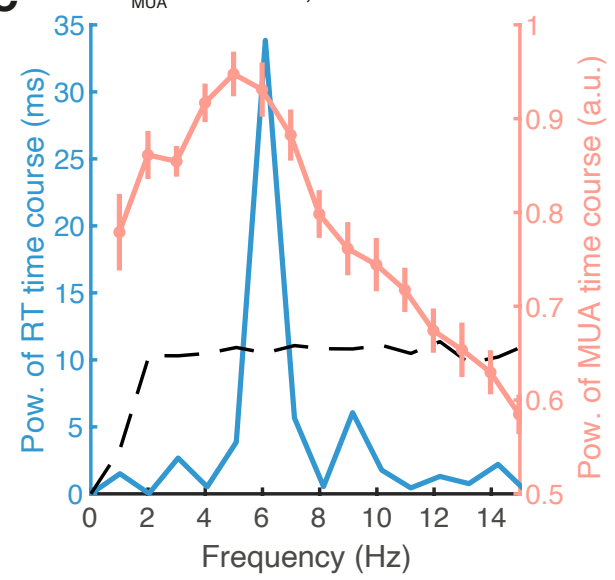

Response
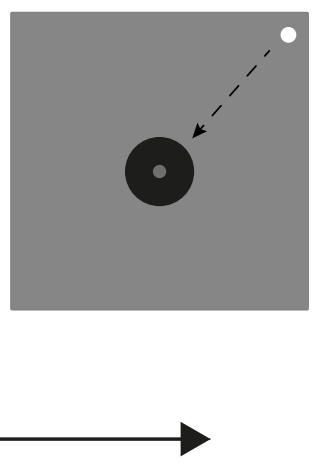

Monkey AL + DP
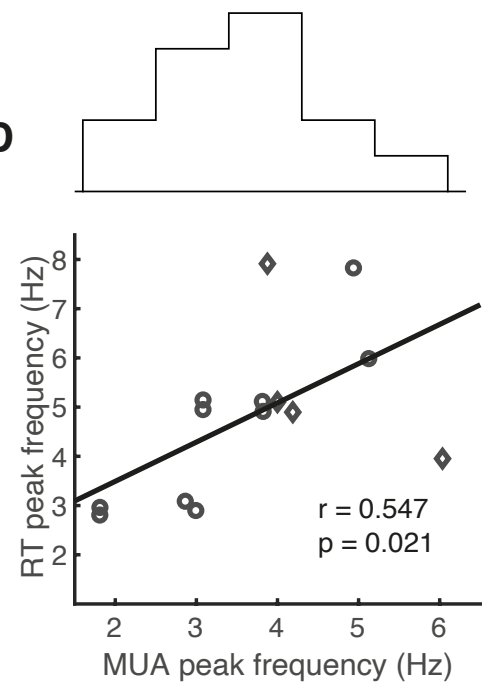

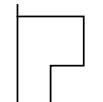

$\mathrm{OAL}$ $\checkmark D P$ 